\title{
Characterization of contrasting rice (Oryza sativa L.) genotypes reveals the Pi-efficient schema for phosphate starvation tolerance
}

\author{
Suresh Kumar ${ }^{1 *}\left(\mathbb{D}\right.$, Pallavi $^{1,2}$, Chetna Chugh ${ }^{1}$, Karishma Seem $^{1}$, Santosh Kumar ${ }^{3}$, K. K. Vinod ${ }^{4}$ and \\ Trilochan Mohapatra ${ }^{5}$
}

\begin{abstract}
Background: Phosphorus (P), being one of the essential components of nucleic acids, cell membranes and enzymes, indispensable for diverse cellular processes like photosynthesis/carbohydrate metabolism, energy production, redox homeostasis and signaling. Crop yield is severely affected due to Phosphate (Pi) deficiency; and to cope with Pi-deficiency, plants have evolved several strategies. Some rice genotypes are compatible with low Pi availability, whereas others are sensitive to Pi deficiency. However, the underlying molecular mechanism for low Pi tolerance remains largely unexplored.
\end{abstract}

Result: Several studies were carried out to understand Pi-deficiency responses in rice at seedling stage, but few of them targeted molecular aspects/responses of Pi-starvation at the advanced stage of growth. To delineate the molecular mechanisms for low Pi tolerance, a pair of contrasting rice (Oryza sativa L.) genotypes [viz. Pusa-44 (Pi-deficiency sensitive) and its near isogenic line (NIL-23, Pi-deficiency tolerant) harboring Phosphorus uptake 1 (Pup 1) QTL from an aus landrace Kasalath] were used. Comparative morphological, physiological, and biochemical analyses confirmed some of the well-known findings. Transcriptome analysis of shoot and root tissues from 45-day-old rice plants grown hydroponically under P-sufficient (16 ppm Pi) or P-starved (0 ppm Pi) medium revealed that Pi-starvation stress causes global transcriptional reprogramming affecting several transcription factors, signaling pathways and other regulatory genes. We could identify several significantly up-regulated genes in roots of NIL-23 under Pi-starvation which might be responsible for the Pi starvation tolerance. Pathway enrichment analysis indicated significant role of certain phosphatases, transporters, transcription factors, carbohydrate metabolism, hormone-signaling, and epigenetic processes in improving P-starvation stress tolerance in NIL-23.

Conclusion: We report the important candidate mechanisms for Pi acquisition/solubilization, recycling, remobilization/transport, sensing/signalling, genetic/epigenetic regulation, and cell wall structural changes to be responsible for P-starvation tolerance in NIL-23. The study provides some of the novel information useful for improving phosphorususe efficiency in rice cultivars.

Keywords: Rice, Phosphorus starvation, Stress tolerance, Transcriptome analysis, Phosphatase, Transporter, Transcription factor, Root development

*Correspondence: sureshkumar3_in@yahoo.co.uk; sureshkumar@iari.res.in ${ }^{1}$ Division of Biochemistry, ICAR-Indian Agricultural Research Institute, New Delhi 110012, India

Full list of author information is available at the end of the article

\section{Background}

Phosphorus (P) is one of the most important macronutrients necessary for the living organisms including plants. It is a vital constituent of several biological 
macromolecules like DNA, RNA and cell membrane, necessary for proper functioning of molecules like enzymes, ATP and NADPH etc., and essentially required for plant growth and development [1]. $\mathrm{P}$ is absorbed by roots mainly in the form of $\mathrm{H}_{2} \mathrm{PO}_{4}{ }^{-}$or $\mathrm{HPO}_{4}{ }^{-2}$ from the soil. Though ample amount of $\mathrm{P}$ is present in soil, its availability in orthophosphate/inorganic phosphate (Pi) form is often hindered due to low solubility, immobility and inaccessibility of $\mathrm{P}$ to plants because of adsorption, precipitation, and/or conversion to organic form [2-4]. Pi-deficiency causes reduced growth of plant, curly leaves, hairy-lateral roots, purple pigmentation in leaves, and/or reduced tillering resulting in severe yield losses [5]. As per an estimate, $30-40 \%$ of the arable land world over has limited crop productivity mainly because of low $\mathrm{Pi}$ content [6]. In India, availability of Pi is low in upland soil, and it is worsening for most of the Indian soils [7]. In fact, Pi-deficiency in soil is becoming a global problem; hence, application of P-fertilizer in soil has become a necessity to ensure better productivity. This is the reason for a considerable increase in the global use of P-fertilizers in crop husbandry. On the contrary, P-use efficiency of crop plants has decreased to as low as $<20 \%$ [8]. However, continuous/extensive use of P-fertilizers would not be economically and ecologically sustainable because of the higher cost of P-fertilizers. Limited stocks of the rock phosphate [9], lower use-efficiency of applied P-fertilizers by crop plants, and excessive application of P-fertilizers lead to the environmental damage [10]. Therefore, the need of the day is to improve P-use efficiency of crop plants, and to explore the possibility of utilizing the naturally available $P$ in the soil. While $P$ acquisition refers to the Pi uptake through roots, P-use efficiency refers to the efficient remobilization/internal use of cellular Pi [11].

Length, number and branching of roots, lateral root/ root-hair density and length (the root morphology), and root angle (root geometry), commonly represented as root system architecture (RSA), play important role in P-uptake from soil [12]. Plant modulates its RSA to increase root surface area for better $\mathrm{P}$ acquisition. Formation of root clusters has been observed in some plant species in response to low $\mathrm{P}$ in soil, which exudate organic acids to acidify/release the chelated ions around the roots, resulting in better availability of $\mathrm{P}$ and other micronutrients [13-15]. Plant releases phosphatase and RNase to enhance $\mathrm{Pi}$ availability and acquisition [16, 17]. Plant possesses specialized transporters and other molecular mechanisms for remobilizing $\mathrm{Pi}$ across the intracellular compartments where the P might have been stored in organic (phytic acid) form. Therefore, efforts are being made to comprehend the mechanisms involved in controlling Pi uptake/homeostasis in plants to improve P-use efficiency [18-20].
Attempts are also being made to analyze transcriptome data for different tissues from various plants, including rice, grown under different conditions until different developmental stages to identify the candidate genes/ mechanisms associated with Pi-deficiency tolerance. However, most of the studies were carried out at the seedling stage [20-25]. Only a limited number of studies used different plant species at advanced stage of development [19-21]. Rice (Oryza sativa L.), one of the most important cereals, is a staple food for more than half of the global population [26]. An aus landrace of rice Kasalath was identified to be tolerant to P-deficiency, which led to the identification of a major quantitative trait loci (QTL) phosphorus uptake 1 (Pup1) mapped on the longer arm of chromosome 12 [27]. Previous efforts to link Kasalath allele at Pup1 with known P-uptake related mechanisms showed that Pup1 near-isogenic lines (NILs) had threefold higher P-uptake efficiency [28]. The Pup1 QTL, generally absent in Japonica and Indica rice (modern) cultivars, has been reported to carry Phosphate Starvation Tolerance 1 (PSTOL1) gene encoding a kinase to enhance Pi acquisition. However, the underlying mechanism for the functions of Pup1 remains enigmatic [29, 30]. Although, no evident P-uptake gene was found to be located on Pup1, the QTL has a larger effect on P-deficiency tolerance [31]. Of the 68 gene models predicted on Pup1, most of them show sequence similarity with transposons, while others could not be annotated with confidence. Some of the genes code for putative fatty acid oxygenase, dirigent-like protein, aspartic proteinase, hypothetical proteins, and putative protein kinase [31].

Transcriptome analysis under P-starvation stress has been performed in many plants including Arabidopsis, rice, wheat, and maize [22, 24, 25, 32-37]. Most of the studies reported differential expression of genes mainly involved in P transport, phosphatases, transcription process, carbon metabolism/photosynthesis, lipid metabolism, cell wall remodeling, etc. Many of these transcriptome studies were conducted with short-duration P-stress only [20, 22, 25, 33-36]. In Arabidopsis, there are nine phosphorus transporter 1 (PHT1) family genes [38], and majority of the $P H T 1$ genes were reported to express in different parts of roots. In rice, 13 PHT1 genes for high-affinity $\mathrm{P}$ transporters were reported to express [39, 40], some of which including OsPHT1;1, OsPHT1;2, OsPHT1;3, OsPHT1;6, and OsPHT1;8 have been functionally characterized [19, 41-43]. A recent study showed that OsPHT1;3 functions in extremely low-P environment to mediate Pi uptake, translocation and remobilization [19], while OsPHT1;11 was reported earlier to be specifically activated by mycorrhizal symbiosis [40]. No significant induction of OsPHT1;1 gene was reported in rice under very low Pi concentration [42]. 
Recently, a SULTR-like phosphorus distribution transporter (SPDT) was reported to play equally important role in distribution of Pi in rice [44].

To cope up with the P-deficiency stress, plants have evolved certain adaptive responses. The phosphorus transporters and related TFs like phosphorus starvation response (e.g. PHR1), SPX (a phosphate-dependent inhibitor of $P H R 1$ ), etc. play crucial roles under P-deficiency stress. OsPHR2 (involved in P-starvation signaling) positively regulates the expression of P-starvation inducible genes, like OsPHTs. Certain other genes have been reported to show variable expressed under $\mathrm{P}$-starvation, which include high-affinity $\mathrm{P}$ transporter (PHT1;6) [41], SPX [45], and monogalactosyl diacylglycerol synthase $(M G D)$ involved in galactolipid synthesis [46]. Moreover, the plant-specific WRKY family TFs have been reported to modulate transcription processes under abiotic stresses [47, 48]. The expression of PHT1 genes was reported to be regulated by TFs due to the presence of cis-acting elements in promoter $[49,50]$. WRKY along with $\mathrm{C} 2 \mathrm{H} 2$ zinc-finger domain containing TFs regulate transcription of target genes [51]. MYB2 functions as transcriptional activator of ABA-dependent/ABA-independent genes under abiotic stresses, and it also activates transcription of miR-399f in Arabidopsis under P-starvation [52]. miR-399 and miR-827 have been reported to positively regulate expression of the genes involved in phosphorus transport and utilization [53].

Phytohormones like auxin, cytokinin, ethylene, and $\mathrm{ABA}$ are involved in transcriptional regulation of the genes for P-starvation responses [54]. ABA-signaling was reported to affect root development, root architecture, root hair density, and root-shoot biomass ratio [55]. Optimum level of gibberellic acid (GA) was reported to be necessary for root-hair growth under P-deficiency. The stress reduces bioactive GA level, which causes accumulation of DELLAs and triggers the responses like alteration in root architecture, reduced shoot growth, and accumulation of anthocyanin in Arabidopsis [56]. P-starvation was reported to up-regulate GA biosynthetic gene GA3-ox2 in myb1 rice mutants, resulting in enhanced GA level and increased length of lateral roots [18]. This demonstrates that MYB1 mediates cross-talk between nutrient signaling and phytohormone signaling pathways. Studies have also reported enhanced expression of jasmonic acid biosynthetic and signaling genes in Arabidopsis and Sorghum [25, 57].

In the present study, comparative transcriptome analysis of shoot and root tissues from 45-day-old plants (at vegetative/tillering stage) of contrasting rice genotypes Pusa-44 (P-starvation sensitive) and NIL-23 (P-starvation tolerant) grown hydroponically under P-sufficient or P-starvation condition revealed some of the candidate genes/mechanisms involved in the stress tolerance. To cope up with Pi-deficiency, plants have evolved a number of strategies including morphological changes like increased length and density of lateral roots, formation of denser and longer root-hairs resulting in better foraging of soil. Biochemical changes like increased production of acid phosphatases and exudation of organic acids, molecular changes like dynamic regulation of gene expression, metabolic changes like reprogramming of carbohydrate and lipid metabolism to help improving P-use efficiency of the plant. Thus, the study provides insights into the differentially expressed genes involved in Pi transport, signaling, phosphatase synthesis, coding for TFs, corehistone domain containing proteins, and glycine-rich cell wall structural proteins to improve P-starvation tolerance in rice. The identified genes/mechanisms for P-starvation tolerance might be utilized in breeding programs to improve yielding potential of rice in P-deficient soils.

\section{Results \\ Morphological and developmental changes in plants under P-starvation}

The contrasting rice (Pusa-44 and NIL-23) genotypes were grown hydroponically in the medium supplemented with/without $\mathrm{Pi}$ till the vegetative/tillering (45 days) stage. We observed significant changes in growth of roots due to P-starvation even at the seedling stage (20-dayold) of plant (Supplementary Fig. S1). Comparison of shoot morphology of the plants grown in hydroponics medium containing varying ( 0 to $40 \mathrm{ppm}$ ) concentration of Pi showed no obvious effects of higher $(20-40 \mathrm{ppm})$ $\mathrm{Pi}$ in the medium. However, lower $\mathrm{Pi}$ concentration $(0-12 \mathrm{ppm})$ in the medium significantly reduced shoot growth compared to the optimum (16 ppm) Pi content (Supplementary Fig. S2). Due to P-starvation stress, a considerable reduction in the growth of shoot and root was observed at vegetative (45 days old) stage of plant (Supplementary Fig. S3). A considerable effect of P-starvation stress was observed on height/biomass of the plants (Fig. 1, Supplementary Table S1). P-starvation/ deficiency caused stunted growth, reduced tillering, and leaves were shorter and erect (Supplementary Fig. S4). At the vegetative stage, a significant reduction in growth of roots, in terms of the number and spread, due to P-starvation under hydroponic conditions was observed (Fig. 2A, B). Moreover, a significant increase in the size of tertiary root/root-hairs was observed due to P-starvation stress in the rice genotypes (Fig. 2C).

\section{Effect on root - shoot biomass ratio}

Comparative analysis of root and shoot biomass produced by the contrasting rice genotypes under P-starvation stress indicated that the stress has considerable 


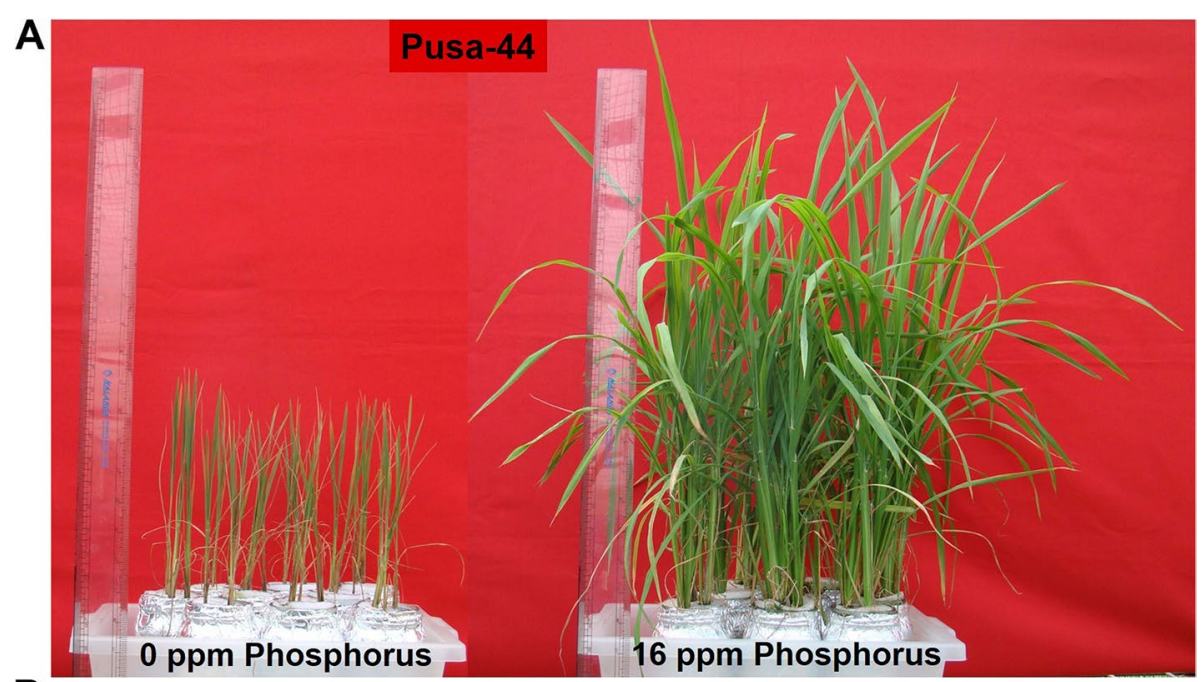

B

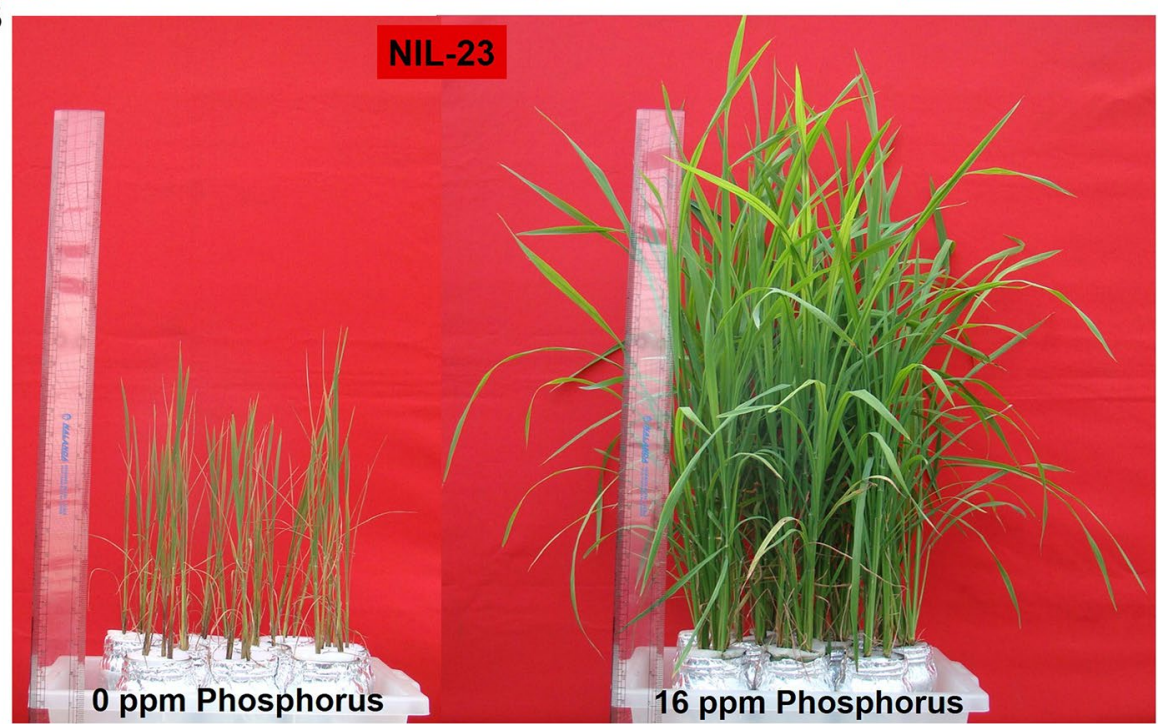

Fig. 1 Shoot morphology of the contrasting rice genotypes. A Pusa-44, stress-sensitive recurrent parent, B near isogenic line (NIL)-23, stress-tolerant grown under Pi-starvation stress. Plants were grown until 45-days under control (16 ppm inorganic phosphorus) or treatment (0 ppm Pi) for comparative assessment

effects on the biomass production. However, the effect of stress was more prominent on shoot biomass production compared to that in root. A considerable reduction (63\%) in shoot biomass was observed due to P-starvation stress. More importantly, the reduction in root biomass was comparatively lesser $(50 \%$ in P-deficiemcy sensitive, and $36-41 \%$ inP-deficiency tolerant genotype) compard to that in shoot. Moreover, root-shoot biomass ratio incrased significantly under the stress in both the stress-sensitive and stresstolerant rice genotypes (Supplementary Table S1). The root - shoot biomass ratio was observed to be higher (0.5) in case of NIL-23 (stress-tolerant), compared to that (0.286) in Pusa-44 (stress-sensitive) genotype.

\section{Effect of P-starvation on leaf morphology and chlorophyll content}

A considerable decrease in size and area of the leaf was observed under P-starvation stress in the rice genotypes. More than 50\% reduction in length and $72 \%$ reduction in width of the leaf was observed in case of P-deficiency sensitive genotype (Pusa-44), while $41-44 \%$ reduction in length and $54-60 \%$ reduction in width was observed in the P-deficiency tolerant (NIL-23 and Kasalath) rice genotypes (Supplementary Fig. S5). Total chlorophyll content in leaf decreased significantly with decreasing availability of $\mathrm{Pi}$ in the hydroponic medium. However, the decrease was considerably higher when $\mathrm{Pi}$ was absent from the medium. 


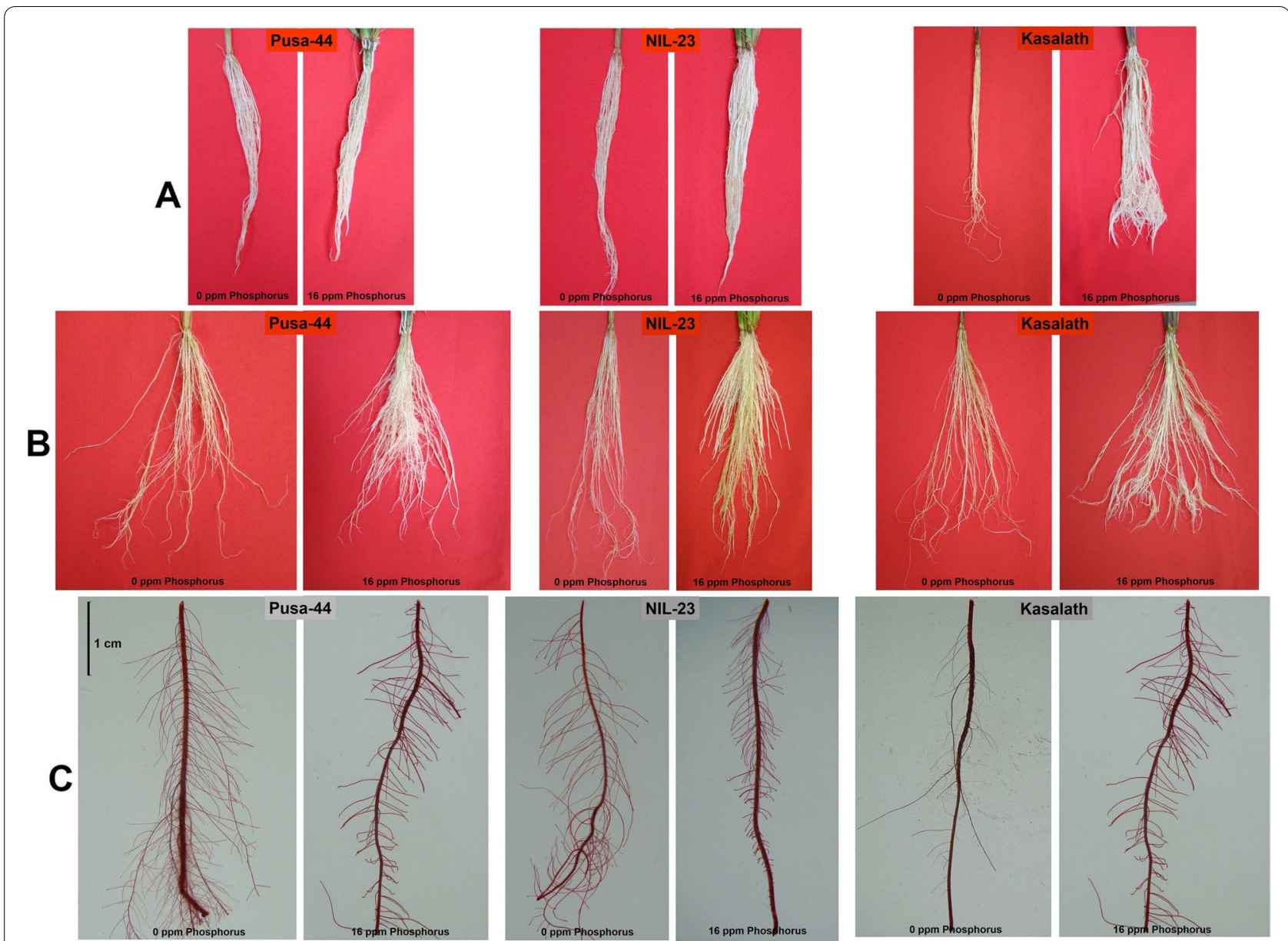

Fig. 2 Root morphology of the contrasting rice genotypes (Pusa-44, stress-sensitive; NIL-23, stress-tolerant) under Pi-starvation stress compared with that of the Pup1 QTL donor (Kasalath). A Comparision of the root bulkiness, B comparision of thickness and spread of the roots, C comparision of the secondary and tertiary roots. Roots of 45-day-old plants grown under control (16 ppm inorganic phosphorus) or treatment (0 ppm Pi) were used for comparative assessment

The response to P-starvation stress was more prominent in Pusa-44 (P-deficiency sensitive) compared to that observed in NIL-23 (P-deficiency tolerant) genotype (Supplementary Fig. S6).

\section{Changes in intrinsic and secreted acid phosphatase activity}

APase activity increased significantly in roots compared to that observed in shoot under P-starvation stress. The increase in APase activity was more prominent in root of the P-deficiency tolerant rice genotypes. With increasing deficiency of phosphorus, the APase activity increased significantly, particularly in the P-deficiency tolerant genotypes. APase activity was also observed to increase in shoot with increasing deficiency of phosphorus in the hydroponic medium (Supplementary Fig. S7).

Secretion of APase from roots was obserbed to increase under P-deficiency stress in all the three rice genotypes. With the increasing deficiency of $\mathrm{P}$ in the hydroponic medium, secretion of APases increased significantly, particularly in P-tolerant rice genotype. However, comparatively lesser secretion of APase was recored from roots when the plants were grown under P-satrvation stress (Supplementary Fig. S8). A significant decrease in $\mathrm{pH}$ of the medium was recorded in presence of $\mathrm{Pi}$ in the medium. The decrease in $\mathrm{pH}$ of the medium was recorded to be more with increasing comcentartion of $\mathrm{Pi}$ in the medium (Supplementary Table S2).

\section{Mobilization of phosphorus in plant tissues}

A significant reduction in $\mathrm{P}$ content was observed with increasing deficiency of $\mathrm{Pi}$ in the hydropnic mdium. Most of the phosphorus acquired by roots is mobilized to shoots through $\mathrm{P}$ transporters, partcularly in case of the P-deficiency tolerant genotypes. Therefore, phosphorus content of shoot was observed to be higher (1.6-2.3fold) than that of the root (Supplementary Fig. S9). The 
ability of P-deficiency tolerant (NIL-23 and Kasalath) genotypes to acquire and accumulate $\mathrm{P}$ was observed to be better. Similarly, mobilization of phosphorus from root to shoot was observed to be better, particularly in case of the tolerant genotypes.

\section{Transcriptome library preparartion, sequencing and mapping on reference genome}

To have comprehensive understanding of the mechanisms involved in Pi-starvation stress tolerance in rice, contrasting rice genotypes for responses to P-deficiency stress were used. A total of 16 libraries for root and shoot tissues from two rice genotypes grown until tillering stage under Pi-sufficient or -deficient conditions were successfully prepared in two replications for whole transcriptome analysis. Total of 395 million reads with an average of 25 million reads for each sample were generated. Reference-based mapping of RNA-seq data on rice reference genome (TIGR v7) using HiSat2 and Stringtie showed $\sim 87 \%$ uniquely mapped reads (Table 1 ).

\section{Differentially expressed genes in contrasting rice genotypes}

To decipher the genes/mechanisms involved in P-starvation stress tolerance in rice, comparative analysis of transcriptome data for root and shoot was performed which resulted in the identification of differentially expressed genes (DEGs) up- or down-regulated based on $\log _{2}$-FC (fold change) and false discovery rate (FDR) $\mathrm{p}<0.05$.
Since the contrasting rice genotypes grown hydroponically in the medium containing 0 or $16 \mathrm{ppm} \mathrm{Pi} \mathrm{were}$ used in the present study, four comparison groups were made for the analysis of DEGs involved in P-starvation tolerance: (i) Roots from Pusa-44 (stress-sensitive genotype) grown in full (16 ppm Pi, control) vs zero (0 ppm $\mathrm{Pi}$, treated). This resulted in identification of $4716 \mathrm{DEGs}$ with 2393 up-regulated and 2323 down-regulated genes under the stress. (ii) Roots from NIL-23 (stress-tolerant genotype) grown in full $\mathrm{Pi}$ (control) vs zero Pi (treated), which resulted in the identification of 4611 DEGs, with 3259 up-regulated and 1352 down-regulated genes under the stress. (iii) Shoots from Pusa-44 grown in full vs zero $\mathrm{Pi}$, showing 2985 DEGs comprising of 1992 up-regulated and 993 down-regulated genes. Similarly, (iv) the comparison of shoots from NIL-23 grown in full vs zero revealed 8515 DEGs including 5627 up-regulated and 2888 down-regulated genes under the P-starvation stress (Fig. 3).

In roots of the stress-tolerant rice genotype (NIL-23), $2402(50.1 \%$ of the DEGs) genes were observed to be exclusively up-regulated, compared to only 1536 (32\%) genes up-regulated in stress-sensitive genotype (Pusa44), along with 857 (17.9\%) commonly up-regulated genes in the contrasting rice genotypes under the stress (Fig. 4A). Among the down-regulated genes, 1856 were observed to be down-regulated in roots of stress-sensitive genotype (Pusa-44) compared to only 885 genes down-regulated in Pusa-44, with 467 (14.6\%) genes

Table 1 Summary oftranscriptome data mapping statistics

\begin{tabular}{|c|c|c|c|c|c|}
\hline Sample ID & Replication & Description $^{a}$ & Total reads & Trimmed reads & $\begin{array}{l}\text { Mapping } \\
\text { efficiency } \\
(\%)\end{array}$ \\
\hline PSF_R1 & 1 & Pusa-44, Shoot, Full (16 ppm) Pi & $23,920,000$ & $22,571,192$ & $95.07 \%$ \\
\hline PSF_R2 & 2 & & $22,765,118$ & $21,575,530$ & $95.02 \%$ \\
\hline PSZ_R1 & 1 & Pusa-44, Shoot, Zero (0 ppm) Pi & $28,000,800$ & $25,864,646$ & $94.04 \%$ \\
\hline PSZ_R2 & 2 & & $27,064,264$ & $25,220,216$ & $94.11 \%$ \\
\hline PRF_R1 & 1 & Pusa-44, Root, Full (16 ppm) Pi & $24,600,000$ & $22,326,862$ & $90.62 \%$ \\
\hline PRF_R2 & 2 & & $24,349,572$ & $22,284,610$ & $90.51 \%$ \\
\hline PRZ_R1 & 1 & Pusa-44, Root, Zero (0 ppm) Pi & $20,400,000$ & $17,204,480$ & $65.70 \%$ \\
\hline PRZ_R2 & 2 & & $20,087,780$ & $16,990,208$ & $65.48 \%$ \\
\hline NSF_R1 & 1 & NIL-23, Shoot, Full (16 ppm) Pi & $24,200,000$ & $22,484,456$ & $94.03 \%$ \\
\hline NSF_R2 & 2 & & $23,828,102$ & $22,263,540$ & $93.93 \%$ \\
\hline NSZ_R1 & 1 & NIL-23, Shoot, Zero (0 ppm) Pi & $28,048,000$ & $26,021,404$ & $89.52 \%$ \\
\hline NSZ_R2 & 2 & & $28,000,318$ & $26,133,560$ & $89.37 \%$ \\
\hline NRF_R1 & 1 & NIL-23, Root, Full (16 ppm) Pi & $26,300,000$ & $24,010,636$ & $88.02 \%$ \\
\hline NRF_R2 & 2 & & $26,105,496$ & $24,097,160$ & $88.21 \%$ \\
\hline NRZ_R1 & 1 & NIL-23, Root, Zero (0 ppm) Pi & $23,800,000$ & $22,380,692$ & $79.68 \%$ \\
\hline NRZ_R2 & 2 & & $23,614,190$ & $22,281,334$ & $79.35 \%$ \\
\hline
\end{tabular}

${ }^{\text {a }}$ The rice plants were grown hydroponically in PusaRicH medium containing full (16 ppm) inorganic phosphorus ( $\mathrm{Pi}$ ) or no/zero (0 ppm) Pi in the medium 


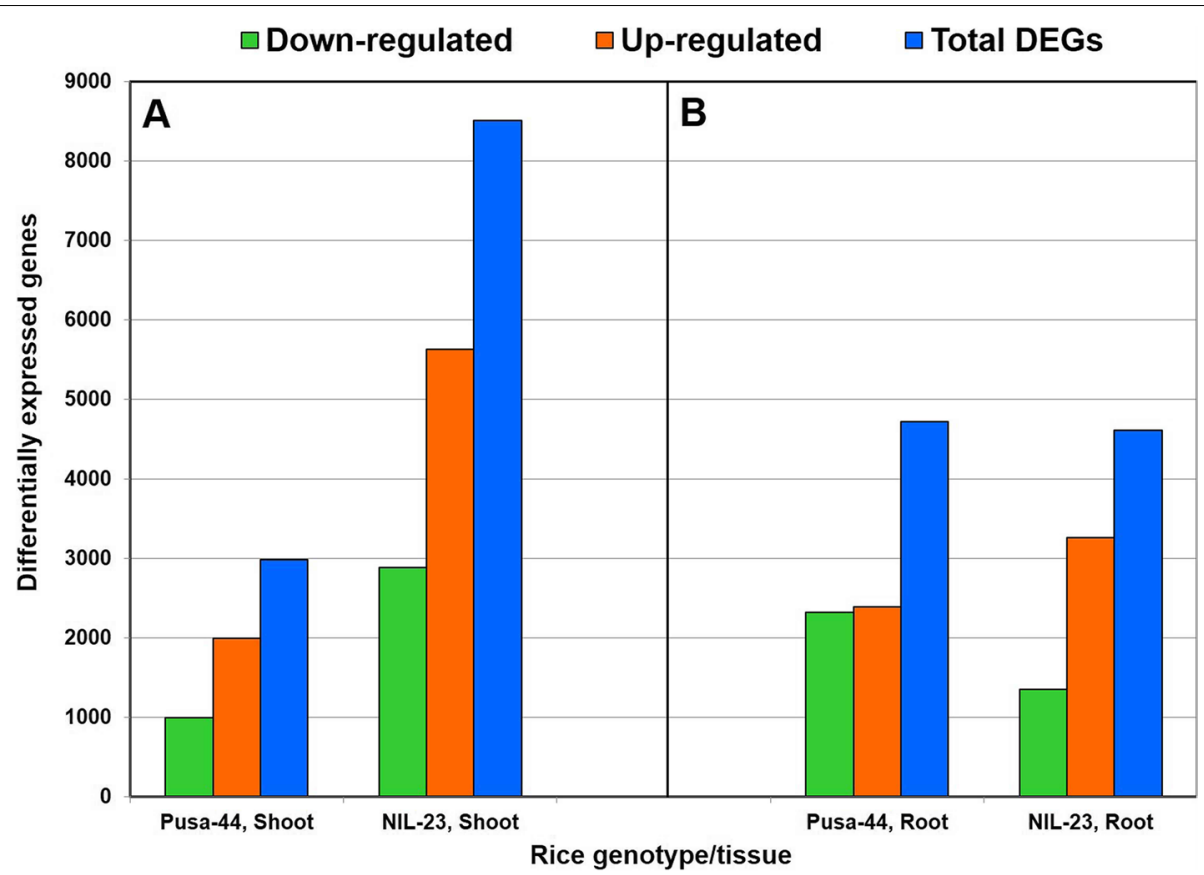

Fig. 3 Differentially expressed genes (DEGs) under P-starvation stress in the stress-sensitive (Pusa-44) and stress-tolerant (NIL-23) rice genotypes grown hydroponically. Shoot and root tissues were collected from 45-day-old plants, continuously grown under control (16 ppm inorganic phosphorus) or treatment (0 ppm Pi) and used for whole transcriptome analysis

A Up-regulated genes in Shoot

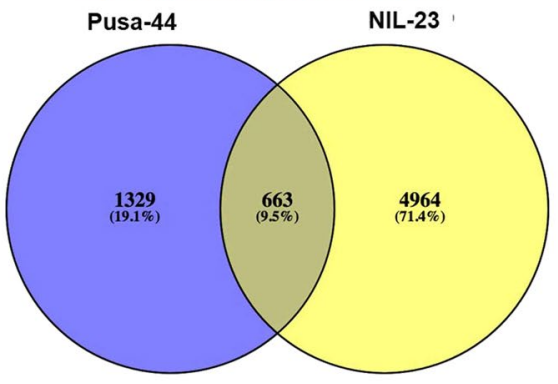

C Down-regulated genes in Shoot

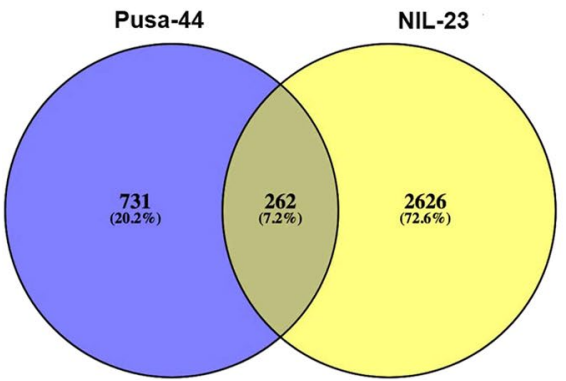

B Up-regulated genes in Root

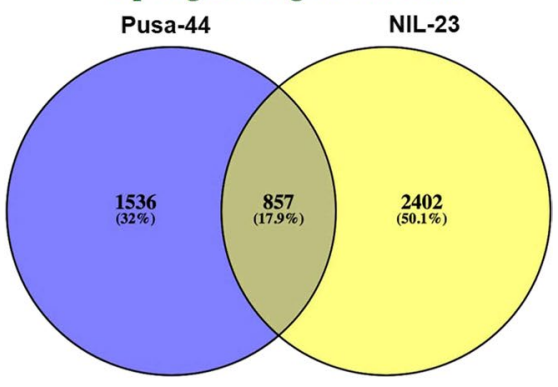

D Down-regulated genes in Root

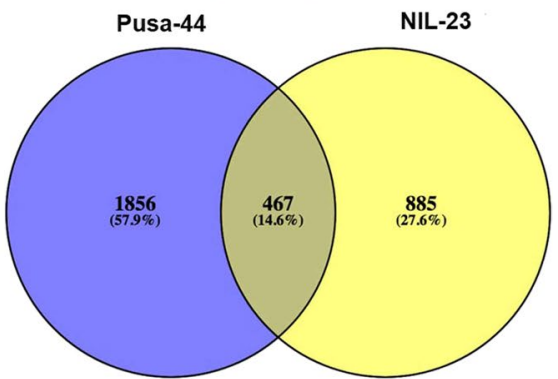

Fig. 4 Differentially expressed genes (DEGs) in stress-sensitive (Pusa-44) and stress-tolerant (NIL-23) rice genotypes grown hydroponically under P-starvation stress. A Differentially and commonly up-regulated genes in shoot of the contrasting rice genotypes, $\mathbf{B}$ differentially and commonly up-regulated genes in root of the contrasting rice genotypes, $\mathbf{C}$ differentially and commonly down-regulated genes in shoot of the contrasting rice genotypes, $\mathbf{D}$ differentially and commonly down-regulated genes in root of the contrasting rice genotypes. Shoot and root tissues were collected from 45-day-old plants, continuously grown under control (16 ppm inorganic phosphorus) or treatment (0 ppm), and used for whole transcriptome analysis 
commonly down-regulated in both the genotypes under the stress (Fig. 4B). However, 262 (7.2\%) genes were commonly down-regulated in shoots of the contrasting rice genotypes due to P-starvation stress.

Similarly, in shoots of the contrasting rice genotypes, $4964(71.4 \%)$ genes were observed to be exclusively upregulated in the stress-tolerant rice genotype compared to only 1329 (19.1\%) genes up-regulated in stress-sensitive genotype, with 663 (9.5\%) genes commonly up-regulated in both the genotypes under the stress (Fig. 4C). Among the down-regulated genes in shoots, 2626 (72.6\%) genes were observed to be down-regulated in root of the stress-sensitive genotype compared to only 731 (20.2\%) genes down-regulated in the stress-sensitive genotype, with 262 (7.2\%) genes commonly down-regulated in both the genotypes under the stress (Fig. 4D). Remarkably, the maximum number of DEGs $(50-71 \%)$ were observed to be up-regulated in shoot and root of the stress-tolerant (NIL-23) genotype under P-starvation stress.

Up-regulated expression of the genes played significant role in stress tolerance, particularly in roots. While $1695(17.1 \%)$ genes were exclusively up-regulated in root of NIL-23, it was only 893 (9\%) in root of Pusa-44. Similarly, 3892 (39.3\%) genes were exclusively up-regulated in shoot of NIL-23, it was only 895 (9\%) in shoot of Pusa44. Moreover, only 367 (3.7\%) and 325 (3.3\%) genes were commonly up-regulated in roots and shoots of both the genotypes, respectively (Fig. 5A).

Down-regulation of the genes played important role in managing stress tolerance in shoot of NIL-23. While 1748 (30.6\%) genes were exclusively down-regulated in shoot of NIL-23, it was only 585 (10.2\%) in shoot of Pusa44. Similarly, 627 (11\%) genes were exclusively downregulated in root of NIL-23, it was only 4467 (20.4\%) in root of Pusa-44. Moreover, only 173 (3\%) and 308 (5.4\%) genes were commonly down-regulated in shoot and root of both the genotypes, respectively (Fig. 5B).

\section{Differential expression of the key phosphorus-responsive genes}

To assess the imposition of P-starvation stress, we tested the expression level of certain P-starvation-inducible genes reported earlier. Our transcriptome data indicated that in root of the NIL-23 69 P-starvation-inducible genes were up-regulated, whereas in root of the stress-sensitive (Pusa-44) genotype 67 genes were upregulated (Supplementary Table S3). Some of the known $\mathrm{Pi}$-starvation-inducible genes, namely phosphoethanolamine/phosphocholine phosphatase (LOC_Os01g52230), purple acid phosphatase (LOC_Os08g17784), glycosyl transferase group 1 domain containing protein (LOC_Os01g04920), phosphoesterase family protein (LOC_Os11g38050), SPX2 domain-containing protein
(LOC_Os03g29250), Ser/Thr protein phosphatase family protein (LOC_Os07g01540), and inorganic phosphate transporter (LOC_Os06g21950), showed $>$ eightfold upregulated expression in root of NIL-23 under the stress. Out of the 76 known Pi-starvation-inducible genes [33], 5 of the genes were expressed in root of NIL-23 only, while 64 genes were commonly expressed in both the genotypes (Fig. 6).

Other genes like soluble inorganic pyrophosphatase (LOC_Os05g02310) and glycosyl hydrolases family 16 (LOC_Os06g48200) showed considerable up-regulation in root of NIL-23 under the stress. Moreover, six genes for Ser/Thr protein phosphatase family protein (LOC_Os07g01540, LOC_Os11g05400, LOC_ Os12g44020, LOC_Os11g34710, LOC_Os03g13540, and LOC_Os07g04210) were observed to be up-regulated in root of NIL-23 during the stress (Supplementary Table S3). Moreover, we observed $>6.3$-fold and $>2.4$ fold up-regulated expression of LOC_Os08g33710 and LOC_Os01g67190 genes, respectively, for ribonuclease T2 family domain containing proteins in roots of NIL-23 under the stress.

\section{Dynamism in expression of P-transporter during P-starvation}

Expressions of Phosphorus-transporters in rice (OsPHTs) showed dynamic variation among the genotypes and tissues. Expressions of six PHT1 genes (OsPHT1;6, OsPHT1;10; OsPHT1;4, OsPHT1;5, OsPHT1;2, OsPHT1;8) were observed to be highly up-regulated and in roots of NIL-23 under P-starvation, whereas OsPHT1;7 showed down-regulated expression (Supplementary Table S4). Interestingly, the fold change in expression of OsPHTs in roots of NIL-23 was more compared to that in root of Pusa-44. OsPHT1;6 showed the highest (12.65fold) level of expression in roots of NIL-23. More importantly, OsPHT1;9 showed exclusive expression (3.52-fold up-regulated) in root of NIL-23. Moreover, OsPHT1;12 expression was observed to be significantly more up-regulated in shoots of the rice genotypes compared to that in roots (Supplementary Table S4), suggesting its role in mobilization of Pi from root to shoot.

\section{Differential expression of transcription factors}

Expression analysis of transcription factors (TFs) in root and shoot of the rice genotypes under P-starvation stress revealed that in root of NIL-23 most of the TFs (210 out of 275) were up-regulated n root. Similarly, in shoot of NIL-23 more number of TFs (277 out of 389) were observed to be up-regulated. Among the differentially expressed TFs, some of the well-known TFs showing significantly up-regulated expression in NIL-23 under the stress include $b Z I P, M Y B, W R K Y$, and $b H L H$ (Fig. 7). 


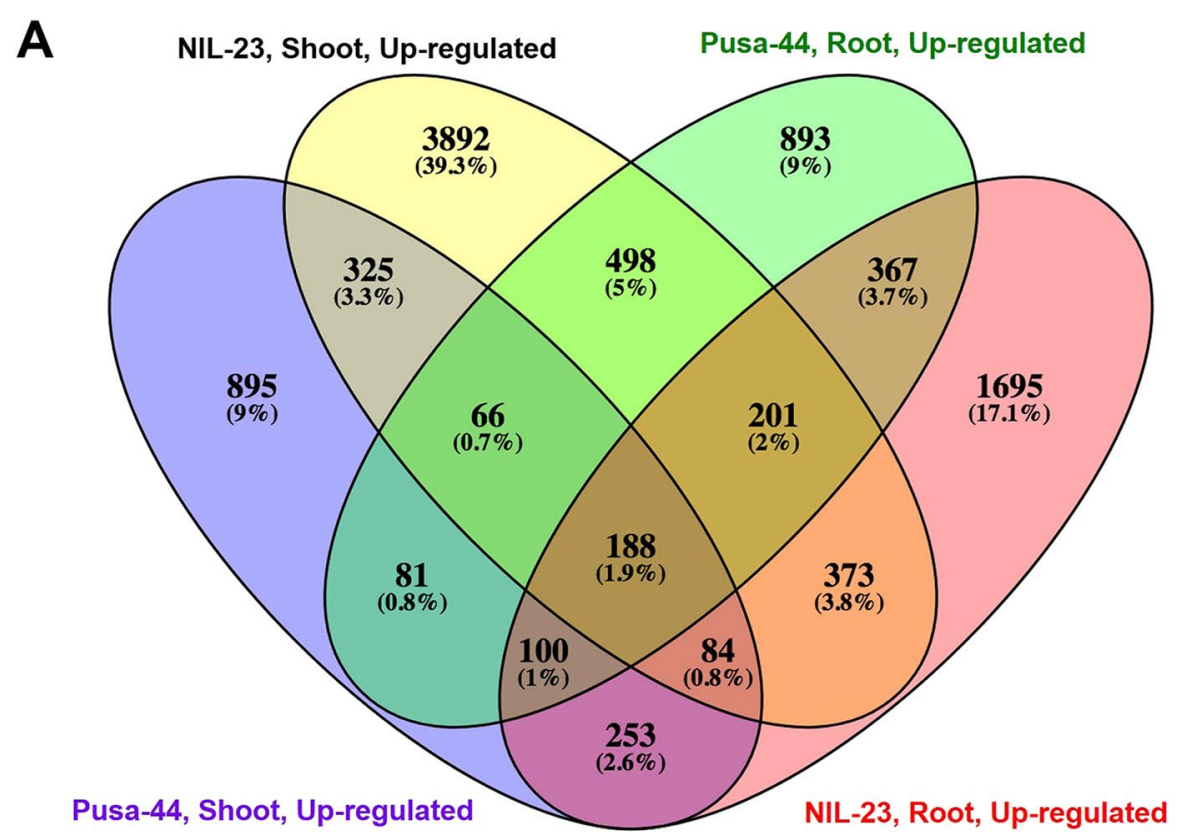

B
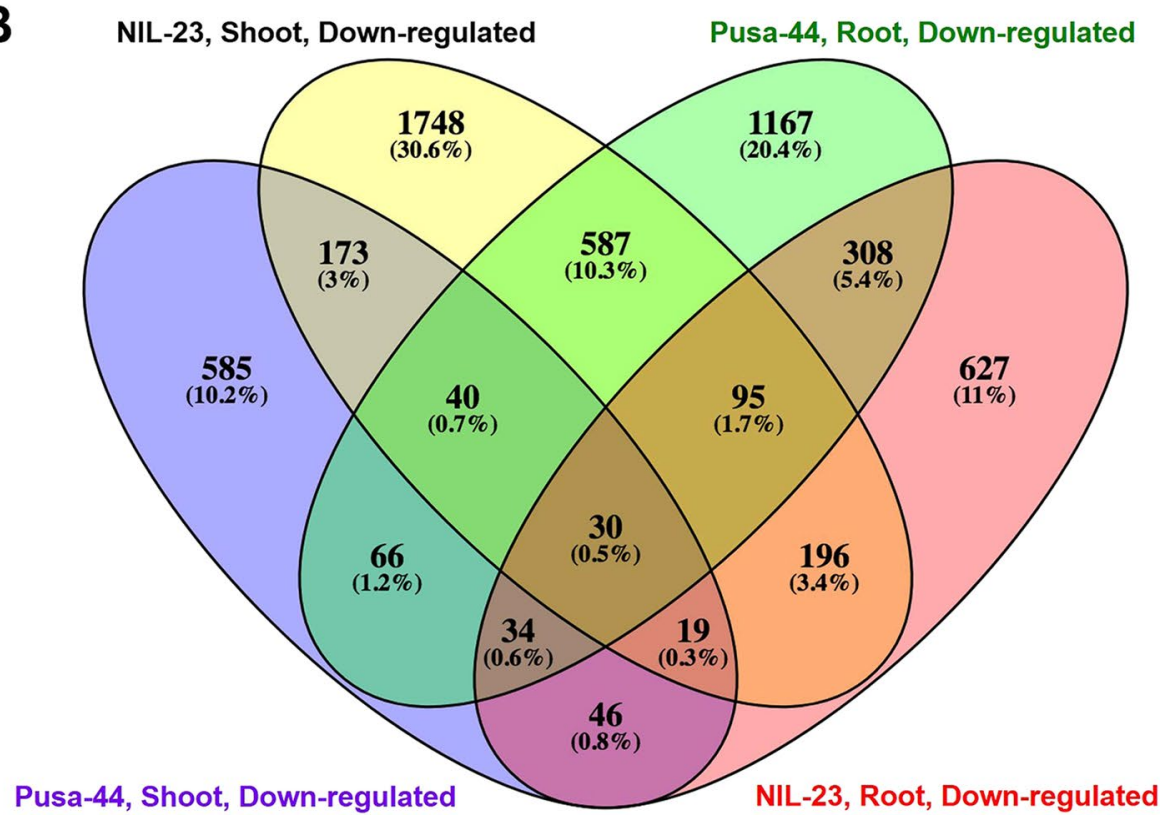

Fig. 5 Four-way analysis of the differentially expressed genes (DEGs) in stress-sensitive (Pusa-44) and stress-tolerant (NIL-23) rice genotypes grown hydroponically under P-starvation stress. A Genotype- and tissue-wise differentially and commonly up-regulated genes in the contrasting rice genotypes, B genotype- and tissue-wise differentially and commonly down-regulated genes in the contrasting rice genotypes. Shoot and root tissues were collected from 45-day-old plants, continuously grown under control (16 ppm inorganic phosphorus) or treatment (0 ppm), and used for whole transcriptome analysis

More interestingly, a homeobox domain containing protein (LOC_Os03g51690) was observed to be considerably up-regulated (8.03-fold) in root and down-regulated (-5.09-fold) in shoot of NIL-23, but its expression was not detected in Pusa-44. Similarly, another homeobox domain containing protein (LOC_Os06g36680), auxin response factor (LOC_Os07g08520), and AP2 domain containing protein (LOC_Os03g15660) were up-regulated in root and shoot of NIL-23. Moreover, the homeobox protein knotted-1 (LOC_Os03g51710) was 


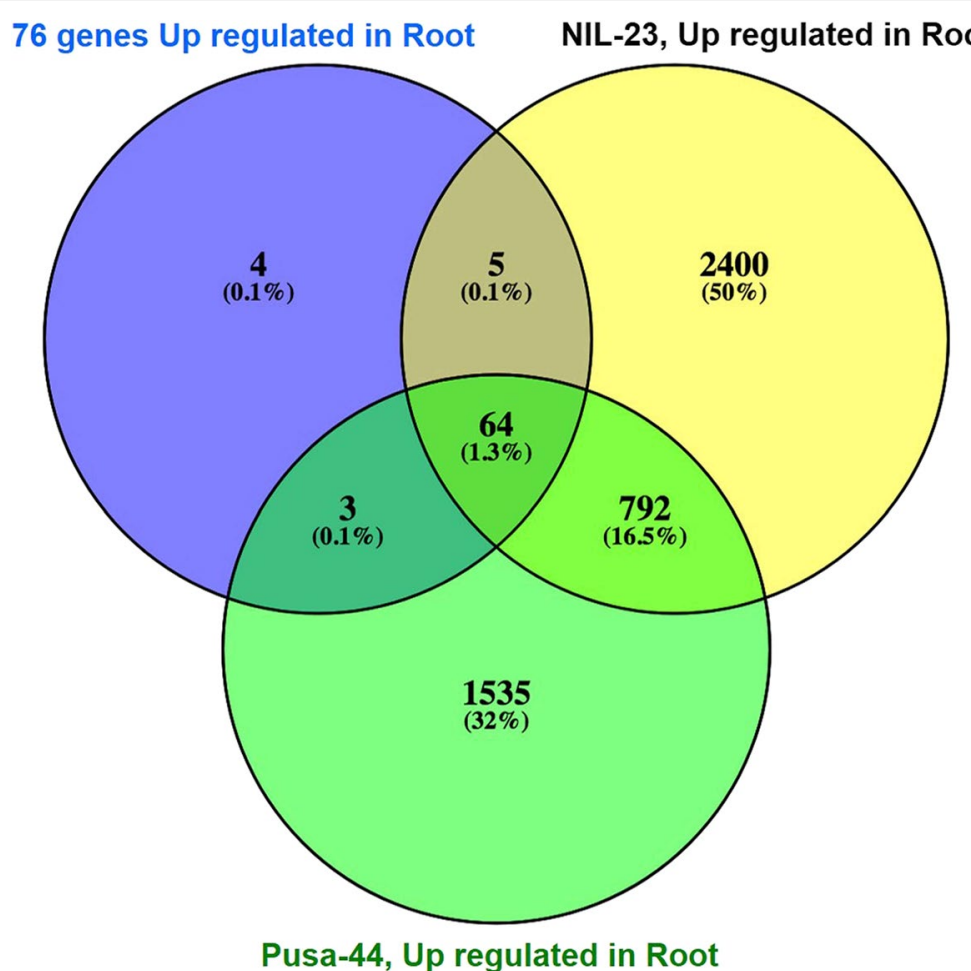

Fig. 6 Expression of the known (76_up_genes_root) Pi-starvation-inducible genes in the stress-sensitive (Pusa-44) and stress-tolerant (NIL-23) rice genotypes grown hydroponically under P-starvation stress. Shoot and root tissues were collected from 45-day-old plants, continuously grown under control (16 ppm inorganic phosphorus) or treatment (0 ppm Pi), and used for whole transcriptome analysis

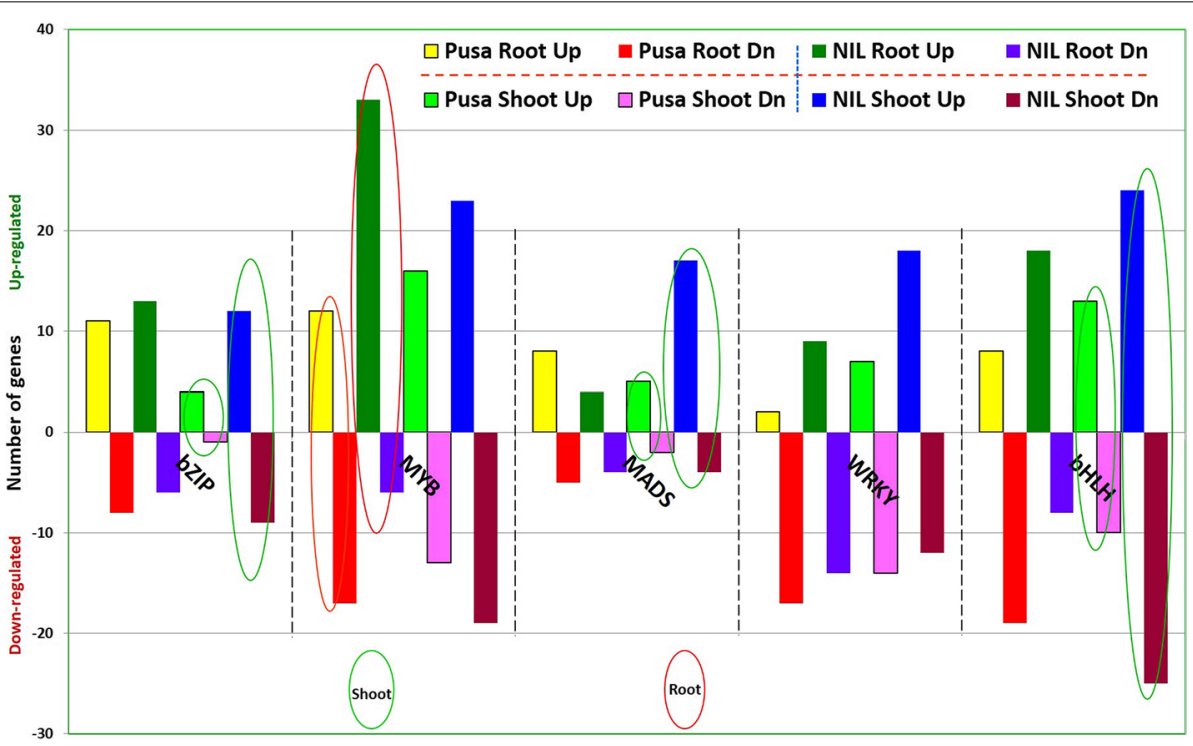

Fig. 7 Differential expression of various transcription factor family genes in shoot and root of the stress-sensitive (Pusa-44) and stress-tolerant (NIL-23) rice genotypes grown hydroponically under P-starvation stress. Shoot and root tissues were collected from 45-day-old plants, continuously grown under control (16 ppm inorganic phosphorus) or treatment (0 ppm Pi), and used for whole transcriptome analysis 
exclusively expressed (6.28-fold up-regulated) in root of NIL-23. Similarly, GRAS family transcription factor containing protein (LOC_Os11g04570), histone-like transcription factor (LOC_Os01g39850), AP2 domain containing protein (LOC_Os04g46410), and HSFtype DNA-binding domain containing protein (LOC Os06g36930) were exclusively expressed ( $>2.5$-fold up-regulated) in root of NIL-23 (Supplementary Table S5).

\section{GO analysis of differentially expressed genes}

To gain insights into the DEGs in the contrasting rice genotypes under the stress, gene ontology (GO) analysis was performed, and their role in diverse biologi$\mathrm{cal} /$ cellular/molecular processes was deciphered. GO analysis for root of NIL-23 indicated a larger number of genes to be up-regulated which were associated with a considerably higher number (229) of GO terms, while smaller number of genes were down-regulated and associated with a lesser number (134) of GO terms. GO analysis for shoot of NIL-23 indicated that up-regulated genes were associated with $131 \mathrm{GO}$ terms, while downregulated genes were associated 199 GO terms. On the other hand, the up- and down-regulated genes in shoot of Pusa-44 were associated 212 and 84 GO terms, respectively.

Under P-starvation stress, the significantly enriched $\mathrm{GO}$ terms belong to molecular functions, suggesting that molecular functions play most important roles in rendering $\mathrm{P}$-starvation stress tolerance. Moreover, biological processes including regulation of gene expression, response to abiotic stress, antioxidant activity, transporter activity, nucleic acid binding TF activity, catalytic activity, and chromatin structure were significantly affected under the stress (Fig. 8). In depth GO analysis of the DEGs revealed dynamic modulation of molecular function, such as phosphate homeostasis, DNA replication, transcriptional/post-transcriptional gene regulation, redox homeostasis, to improve the stress tolerance (Fig. 9). In addition, the genes involved in carbohydrate and lipid metabolism, nitrogen metabolic processes, photosynthesis, were observed to be modulated by the stress. Epigenetic regulation of gene expression and post-translational protein modification were the other important GO terms over-represented.

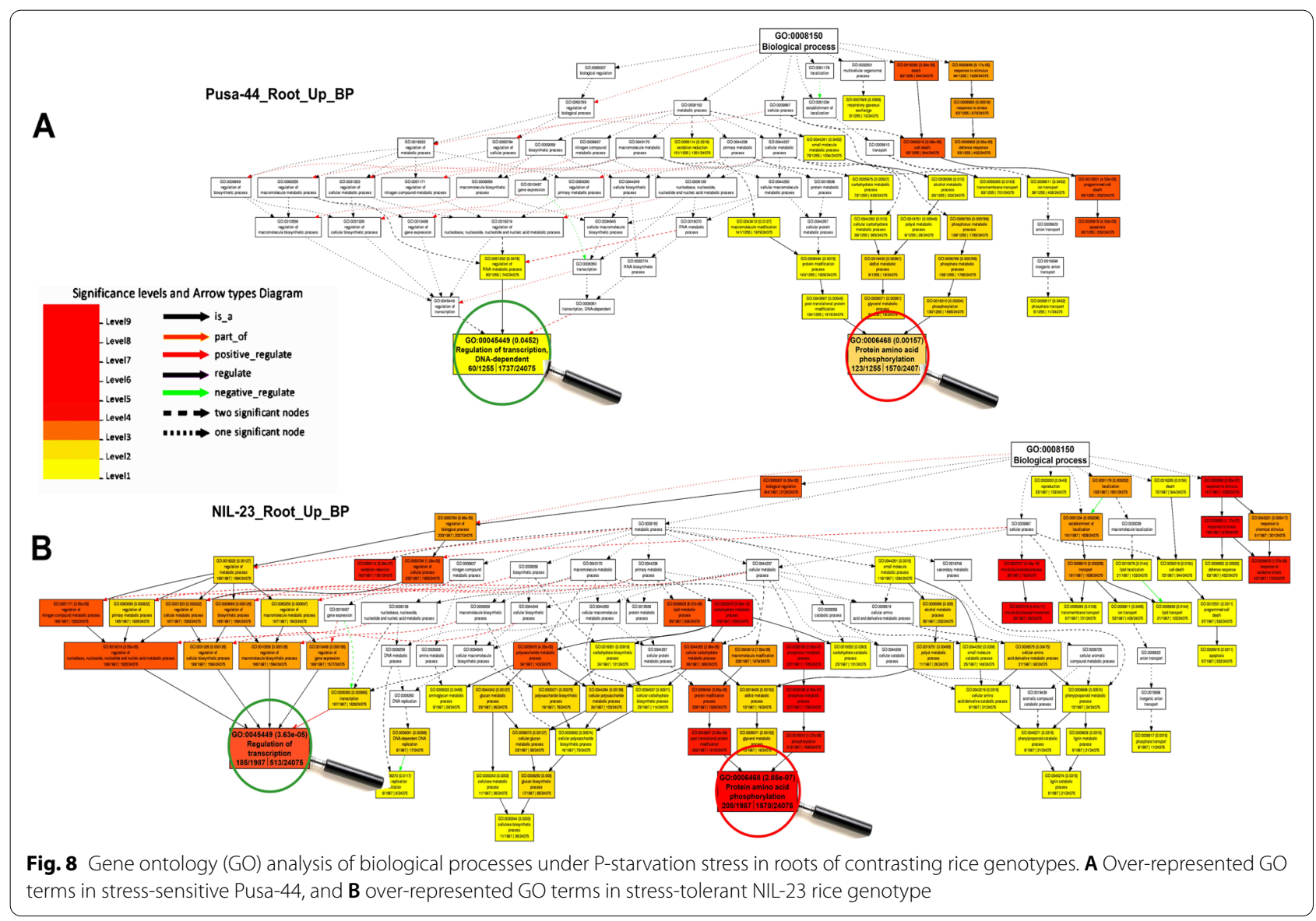




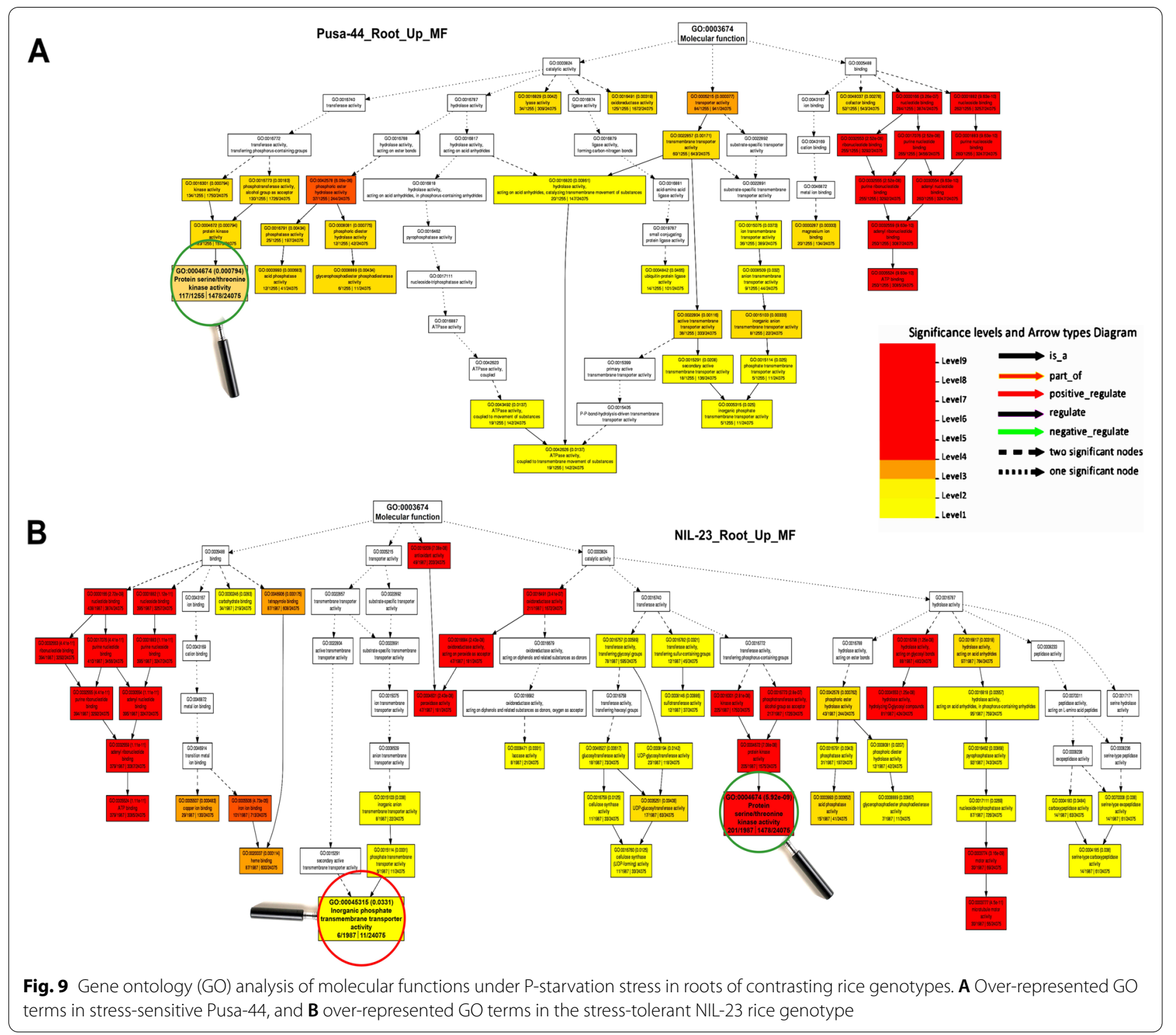

\section{Differential expression of cell wall associated genes}

In depth analysis of the DEGs suggested that a subset of genes are involved in cell wall and associated activities during P-starvation stress. Such genes include glycinerich cell wall structural protein and expansins that showed considerably up-regulated expression in root of NIL-23 during the stress (Supplemental Table S6). The stress caused up-regulated ( sevenfold) expressions of glycinerich cell wall structural protein gene (LOC_Os10g31540) in roots of NIL-23, while it was down-regulated ( threefold) in roots of Pusa-44. Expression of expansin precursor gene (LOC_Os10g40730) was up-regulated $(\sim 3.8$-fold $)$ in roots of NIL-23, while down-regulated $(\sim 2.5$-fold $)$ in roots of Pusa-44. Similarly, expressions of CESA7-cellulose synthase (LOC_Os10g32980) was up-regulated $(\sim 4.6$-fold) in roots of NIL, while downregulated ( 1.4 -fold) in roots of Pusa-44. In addition, several other genes, including glucan endo-1,3-betaglucosidase precursor (LOC_Os07g35520), microtubule associated protein (LOC_Os01g49200), extra-large G-protein-related (LOC_Os02g15820) and tetraspanin family protein (LOC_Os06g44310), were observed to be up-regulated in roots of NIL-23, but down-regulated in roots of Pusa- 44.

Some of the LTPL-Protease inhibitor family proteins (e.g. LTPL143, LOC_Os10g40480; LTPL139, LOC_ Os10g40430) were observed to be considerably (4 to eightfold) up-regulated in roots of NIL-23, whereas these were down-regulated ( 2 to eightfold) in roots of Pusa44 under P-starvation stress. Moreover, expressions of 
aquaporin (LOC_Os03g05290) and ABC transporter, ATP-binding protein (LOC_Os05g04610) were also observed to be up-regulated in roots of NIL-23, whereas down-regulated in roots of Pusa-44 under the stress (Supplementary Table S6).

\section{Differential expression of genes for phytohormone and signal transduction}

Expression of auxin-responsive protein (LOC_ Os05g48270) was observed to be up-regulated $(\sim 5.82$-fold $)$ in roots of NIL-23, while down-regulated $(\sim 2.13$-fold $)$ in Pusa-44 under P-starvation stress. Similarly, an auxin-induced protein 5NG4 (LOC_Os01g36580) was observed to be highly (5.69-fold) up-regulated in roots of NIL-23, but down-regulated in roots of Pusa-44. Other auxin-responsive family genes (like LOC_Os12g40900, LOC_Os12g40890, LOC_ Os02g05050, LOC_Os02g13520) were also observed to be up-regulated in roots of NIL-23 but down-regulated in Pusa-44 under the stress (Supplementary Table S7). Moreover, the genes for auxin efflux carrier component (e.g. LOC_Os06g44970, LOC_Os02g50960) were also observed to be up-regulated in roots of NIL-23, whereas down-regulated in roots of Pusa-44 under P-starvation stress.

Expression of the genes involved in synthesis or response to other phytohormones, like jasmonateinduced protein (LOC_Os04g22900) and gibberellin 2 - $\beta$-dioxygenase (LOC_Os05g48700) were also observed to be up-regulated ( $\sim 5.98$ and $\sim 4.52$-fold, respectively) in roots of NIL-23, but down-regulated in roots of Pusa44 under P-starvation stress (Supplementary Table S7).

\section{Differential expression of genes involved in carbohydrate and lipid metabolism}

Some of the genes involved in carbohydrate and lipid metabolism were observed to be differentially expressed in root of the contrasting rice genotypes. The gene for glycerophosphoryl diester phosphodiesterase (LOC_ Os03g40670) associated with cell wall organization was observed to be up-regulated in roots under P-starvation stress, particularly in NIL-23. Other genes like glycosyl hydrolase (LOC_Os05g15770), lactate/malate dehydrogenase (LOC_Os08g33720), enolase (LOC_Os03g14450), phosphoenolpyruvate carboxylase (LOC_Os08g27840), pyruvate kinase (LOC_Os11g05110), glyceraldehyde3-phosphate dehydrogenase (LOC_Os08g03290) were also observed to be up-regulated in roots of NIL-23 under the stress. However, genes for trehalose-6-phosphate synthase (LOC_Os09g20990), sucrose synthase (LOC_Os06g09450), and asparate aminotransferase (LOC_Os09g28050) were observed to be down-regulated in roots of the contrasting rice genotypes under the stress (Supplementary Table S8).

\section{Differential expression of photosynthesis related genes}

Expression level of the gene for photosystem II (44 KDa) reaction center (LOC_Os04g16874) was observed to be down-regulated in root of both the rice genotypes, particularly in NIL-23 (-5.95-fold) under P-starvation stress. Similarly, oxygen-evolving enhancer protein 1 (LOC_Os01g31690), cytochrome b6 (LOC_Os10g21324), and ATP synthase subunit alpha (LOC_Os04g16740) were also observed to be down-regulated in roots of the rice genotypes under the stress. Genes for the other components of photosynthesis machinery, like NADPHdependent oxidoreductase (LOC_Os10g21418), chlorophyll A-B binding protein (LOC_Os06g21590), and photosystem II P680 chlorophyll A apoprotein (LOC_ Os10g21310), were also observed to be significantly down-regulated in root tissues of both the rice genotypes. (Supplementary Table S9). On the other hand, the genes for photosystem II P680 chlorophyll A apoprotein (LOC_ Os10g21310) and cytochrome b6 (LOC_Os10g21324) were observed to be up-regulated in shoot of NIL-23 under the stress.

\section{Differential expression of genes involved in epigenetic regulation}

Many of the genes involved in epigenetic regulation of gene expression were observed to be differentially expressed. Some of the important genes involved in histone-modification include core histone $\mathrm{H} 2 \mathrm{~A} / \mathrm{H} 2 \mathrm{~B} /$ $\mathrm{H} 3 / \mathrm{H} 4$ domain containing protein (LOC_Os10g28230, LOC_Os03g06670, LOC_Os03g02780) were up-regulated in root of NIL-23 (stress tolerant genotype), down-regulated in Pusa-44 (stress sensitive genotype) under P-starvation stress (Supplementary Table S10). Moreover, the gene for $\mathrm{jmjC}$ domain containing protein (LOC_Os02g58210), involved in histone-demethylation, was observed to be 1.44-fold up-regulated in roots of NIL-23 under the stress, while it was down-regulated (-1.74-fold) in Pusa-44 rice genotype. Expression of C-5 cytosine-specific DNA methylase (LOC_Os10g01570) was observed to be up-regulated $(\sim 3.33$-fold $)$ in roots of the NI-23 under the stress, whereas it was downregulated (2.48-fold) in roots of Pusa-44. Moreover, the gene for methyl-CpG binding domain containing protein (LOC_Os12g42550) was observed to be down-regulated in roots of the stress tolerant rice genotype. Interestingly, the genes for hsp20- $\alpha$-crystallin family protein (LOC_ Os03g06170, LOC_Os10g07210, LOC_Os10g07200), low-molecular-weight $(20 \mathrm{kDa})$ protein produced under heat-shock or other environmental stress, were observed to be up-regulated (2.8 to 5.5 -fold) in roots of NIL-23 
under the stress, while they were down-regulated (2.9 to 3.9-fold) in roots of Pusa-44 (Supplementary Table S10).

Validation of transcriptome data by Real-Time PCR analysis To validate the DEGs in the contrasting rice genotypes, expression level of seven randomly selected genes was validated in root and shoot in response to P-starvation stress by RT-qPCR analysis. The results were consistent with the expression pattern of the genes detected by RNA-seq. (Fig. 10). Thus, the RT-qPCR results confirmed trustworthy of the data we obtained from RNA-seq.

\section{Discussion}

Inorganic phosphorus is often a limiting factor for plant growth, development, and productivity. However, plants take adaptive measures like altering root growth/architecture to access a larger volume of soil in search of $\mathrm{P}$, excretion of organic acids/phosphatases/nucleases to solubilize Pi from organic sources and/or recycle internal Pi [2] to mitigate the effects of stress. Though some of the components of P-deficiency signaling in plants have been identified, the major pathways are still unexplored. PSTOL1, coding for a Pup1-specific protein kinase [29], was identified to impart variation in P-deficiency tolerance among the modern sensitive rice varieties and the tolerant (Kasalath) genotype. Most of the investigations on P-deficiency tolerance in plants were carried out at the seedling stage $[20-25,58]$ as plant-stand establishment is the first and one of the important steps for crop productivity. Once the seedlings are established, requirement of $\mathrm{P}$ for the growth and development (vegetative growth) of the plants is equally important. During the vegetative stage, plants grow rapidly and the requirement of $\mathrm{P}$ is considerably higher than that required during seedling stage. Hence, sevearl biochemical, physiological and molecular strategies are adopted by the plants to meet the requirements [59].

Therefore, we aimed at investigating the strategies/ mechanisms adopted by rice plants to cope up with the limited availability of $\mathrm{Pi}$ beyond the seedling stage of growth. When a plant grows in P-deficient soil, it faces a continueous stress starting from germination through tillering until maturity. Hence, the rice plants were grown under P-starvation stress starting from the germination. Moreover, zero Pi in the hydroponic medium provides the most contrasting condition with respect to the concentration of $\mathrm{Pi}(16 \mathrm{ppm})$ supplied, which has been reported to be the best $\mathrm{Pi}$ concentration in PusaRicH medium for growth of a large number of rice genotypes in hydroponics [60]. Therefore, we used no/zero (0 ppm) $\mathrm{Pi}$ in the hydroponic medium for the stress treatment.
Morphophysiological and biochemical adaptations in plant The comparative morphological analyses of rice plants grown in P-sufficient and P-starvation conditions indicated a significant reduction in the number of roots but considerable increase in the length at seedling stage (20-day-old) of plant due to P-starvation. However, the tolerant genotypes showed more $(50-60 \%)$ increase in the root length (Supplementary Fig. S1), which is in agreement with the earlier findings in different plant species $[11,25,28,35,61]$. This enables better $\mathrm{Pi}$ acquisition by increasing the root surface area for $\mathrm{P}$ absorption under the stress. Based on a pilot experiment, indicating no significant improvement in plant morphology with a higher than $16 \mathrm{ppm}$ Pi concentration in the medium (Supplementary Fig. S2), the upper limit of Pi in the hydroponic medium was determined. Hence, 16 ppm Pi in PusaRicH medium was used to grow the rice plants under control condition. Further, a significant effect of P-starvation was observed on root and shoot development in all the three rice genotypes (Supplementary Fig. S3).

As $\mathrm{P}$ is one of the essential nutrients for living organisms, required for nucleic acids and phospholipids biosynthesis, enzyme activity, signal transduction, energy transport in the form of ATP, and several other metabolic processes, P-starvation considerably affects root and shoot growth in terms of reduced biomass production. At tillering stage (45-day-old plants), $71 \%$ reduction in height of Pusa-44 plants was observed when grown under P-starvation (0 ppm Pi) stress compared to that in the plants grown under control condition. Such reduction in height of NIL-23 and Kasalath plants was recorded to be only 47 and $51 \%$, respectively (Fig. 1, Supplementary Fig. S4). Leaf being one of the most important parts of the plant for growth and development, P-starvation caused considerable reduction in size $(51 \%$ reduction in stresssensitive genotype, and $41-44 \%$ reduction in the stresstolerant genotypes) as well as surface area ( $84 \%$ reduction in stress-sensitive genotype, and $68-70 \%$ reduction in the stress-tolerant genotypes) of the leaf (Supplementary Fig. S5). As expected, we observed a significant reduction in chlorophyll content with decreasing availability of $\mathrm{Pi}$ in the hydroponic medium. However, the reduction was considerably higher when Pi was not present in the medium (Supplementary Fig. S6). P-deficiency was reported to affect chlorophyll content in leaf [62]. Considering the importance, chlorophyll $a$ fluorescence analysis was proposed to be used to detect P-deficiency in soil under field condition [63].

For a more comprehensive understanding, the effect of P-starvation on growth of root, shoot and root-shoot biomass ratio was analyzed. This revealed a significant effect of P-starvation on growth of root (number/biomass, spread, and branching/tertiary root/root-hairs), 


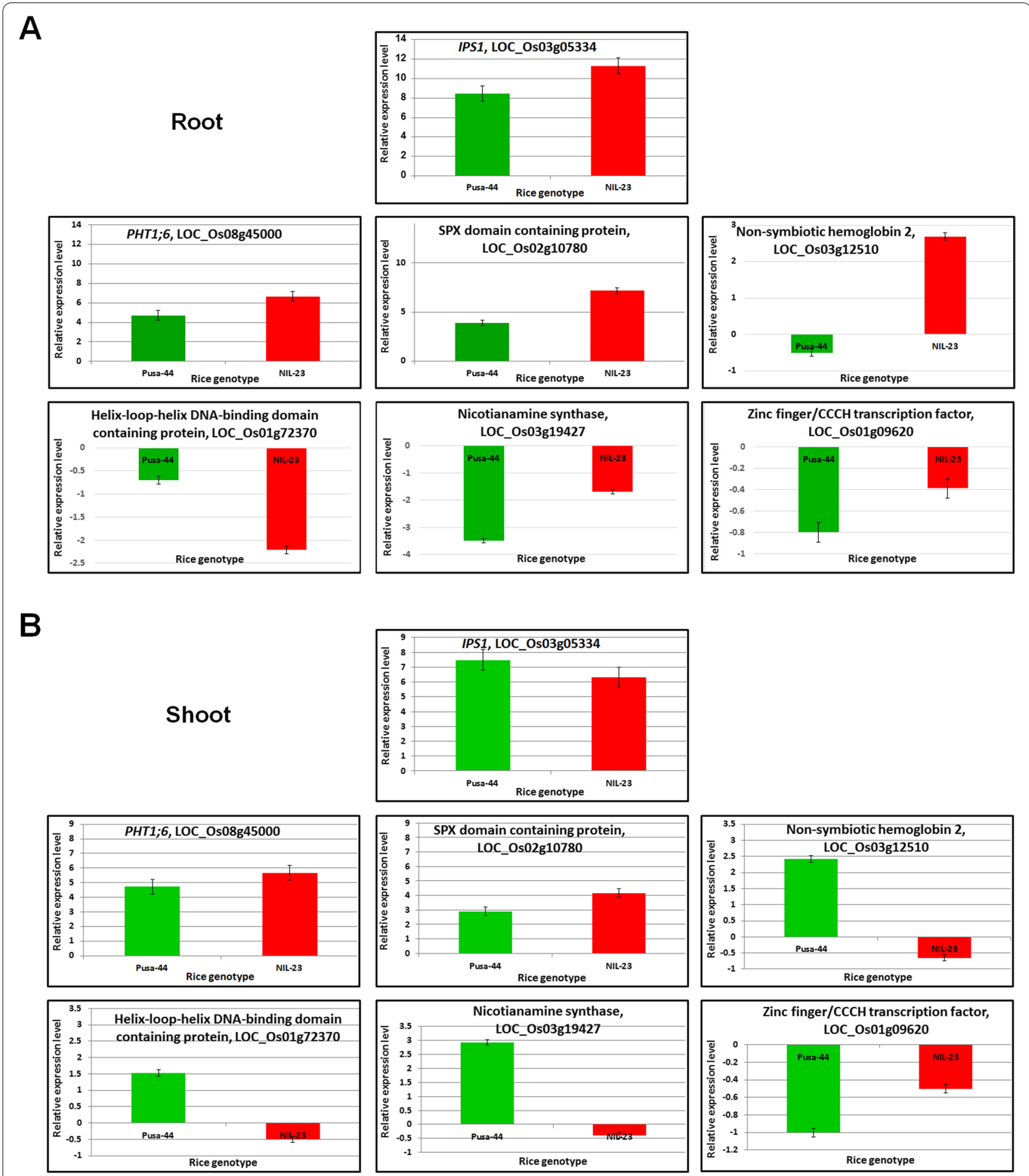

Fig. 10 RT-qPCR validation of seven randomly selected differentially expressed genes. cDNA was prepared for the tissues collected from 45-day-old plants of P-deficiency stress-sensitive (Pusa-44) and stress-tolerant (NIL-23) rice genotypes grown hydroponically under control (16 ppm inorganic phosphorus) or treatment (0 ppm Pi). Data represent the mean $\pm S D(n=3)$ 
particularly in Pusa-44 (Fig. 2, Supplementary Table S1). Plants deploy several strategies to acquire $\mathrm{P}$, with emphasis on exploration and uptake of Pi from soil [64]. This is generally achieved by increasing root growth, which results in increased root - shoot biomass ratio. However, the increased root growth requires a large proportion of daily photosynthate, which may be further constrained due to the limited supply of $\mathrm{Pi}$ [64]. Our findings are in agreement with those of Péret et al. [65]. The increased root - shoot biomass ratio has been reported in tomato, onion maize, and sorghum [24, 25, 65, 66]. Moreover, our observations on increased root length and tertiary roots/ root-hairs corroborate with the earlier findings [25, 28]. However, a significant reduction in spread of roots was observed due to P-starvation stress (Fig. 2B). The length of tertiary roots/root-hairs increased significantly due to the stress (Fig. 2C), which helps improving P acquisition by the plants. Nguyen and Stangoulis [61] proposed a change in RSA to be an indicator of P-deficiency tolerance in wheat. In certain plant species, root-cluster (proteoid roots) formation has also been reported in response to P-deficiency. Such specialized roots secrete organic acids, which acidify the soil and chelate metal ions around the roots [13]. This might be one of the reasons for the observed reduction in $\mathrm{pH}$ of the hydroponic medium (Supplementary Table S2).

A considerable increase in the production of acid phosphatases in roots (Supplementary Figure S7) and their secretion in the soil were observed, which help mobilizing the P present in fixed forms. Phosphatases, ribonucleases and organic acids release Pi from organic/inorganic compounds in the rhizosphere. The up-regulated expression of phosphocholine phosphatase (Os01g52230) and ser/thr phosphatase (Os10g02750) genes under P-starvation corroborates with findings of Mehra et al. [11]. Increase in APase activity was observed in roots, which increased with the decreasing Pi content in the hydroponic medium. Increase in APase activity was reported earlier by Yugandhar et al. [67] in rice under P-deficiency stress. Up-regulated expression of the genes coding for enzymes/proteins responsible for organic acid synthesis and exudation under P-deficiency stress has been reported earlier [68-70]. Moreover, the observed up-regulated expression of the genes involved in glycolysis is necessary to increase carbon supply for organic acid synthesis. Our observation on $>6.3$-fold and $>2.4$ fold up-regulated expression of LOC_Os08g33710 and LOC_Os01g67190 (the ribonuclease T2 family domain containing proteins), respectively, is in agreement with the findings of Gho et al. [17]. Increased excretion of organic acids and Pi-releasing enzymes such as RNases and purple acid phosphatases (PAPs) results in larger rhizospheric Pi pool for uptake. The increased roots/ root-hairs enhance porosity and oxygen release by the plant resulting in oxidation of iron and release of protons, which cause increased rhizospheric acidity to solubilize soil P. Secretion of purple acid phosphatases (PAPs), like AtPAP10, AtPAP12, PvPAP1 and PvPAP3 has also been reported earlier to help releasing $\mathrm{Pi}$ and organic $\mathrm{P}$ like ADP, glycerol-3-P, dNTPs in Arabidopsis and Phaseolus vulgaris [71]. Besides, overexpression of WRKY1 from Gossypium barbadense in Arabidopsis was reported to increase accumulation of acid phosphatases, the number of lateral roots, and P-content in root [72].

P-content in plant tissues was observed to increase with increasing availability of $\mathrm{Pi}$ in the hydroponic medium (Supplementary Fig. S9). More P in shoot compared to that in root, and higher $\mathrm{P}$ content with increasing $\mathrm{Pi}$ in the medium are in agreement with the findings reported by Yugandhar et al. [65] in rice. We observed better $\mathrm{P}$ acquisition by roots of the stress-tolerant genotypes even at lower $\mathrm{P}$ in the hydroponic medium. This resulted in higher P content in root and shoot of NIL-23 and Kasalath (stress-tolerant genotypes) compared to that in Pusa-44 (stress-sensitive genotype). Thus, these morphophysiological and biochemical traits are influenced by environment and controlled by various molecular regulators, which interact synergistically to increase $\mathrm{P}$ acquisition [73]. For example, our molecular data clearly indicate that the key genes involved in glycolytic bypasses were significantly up-regulated in NIL-23; thus helped manage the demand of Pi very efficiently even under P-starvation stress in association with other synergistic strategies.

\section{Molecular adaptations under P-starvation}

In addition to the morphological, physiological and biochemical adaptations, plants implement molecular approaches to cope up with the P-deficiency stress by modulating gene expression. DEGs analysis in root and shoot tissues of the contrasting rice genotypes in response to P-starvation stress revealed that up-regulation of gene expression play major role in stress tolerance. The number of up-regulated genes was comparatively higher in both shoot and root of the P-deficiency tolerant (NIL-23) genotype (Fig. 3). With a similar number of total DEGs, significantly higher number of up-regulated genes and lower number of down-regulated genes in root of NIL-23, compared to that in Pusa-44, might be responsible for the observed $\mathrm{P}$-starvation tolerance in NIL-23. Moreover, considerable increase in the number of up-regulated as well as down-regulated genes in shoot of NIL-23 helps managing growth of plant under P-starvation stress. The uniquely up-regulated (2402) genes in root of NIL-23 were $~ 1.6$ times more than that in Pusa44. Similarly, the uniquely up-regulated (4964) genes 
in shoot of NIL-23 were $\sim 3.7$ times more than that in Pusa-44. More importantly, the uniquely down-regulated genes (2626) in shoot of NIL-23 were 3.6 times higher than that in the shoot of Pusa-44 (Fig. 4). The exclusively up-regulated (1695) genes in root of NIL-23 were 1.9 times higher than that in root of Pusa-44. Similarly, the exclusively up-regulated (3892) genes in shoot of NIL23 were $\sim 4.3$ times higher than that in shoot of Pusa-44 (Fig. 5A). The exclusively down-regulated (1743) genes in shoot of NIL-23 were observed to be $\sim 3$ times higher than that in shoot of Pusa-44 (Fig. 5B). interestingly, out of the 76 phosphorus-responsive marker genes identified from earlier studies in Arabidopsis and rice [33], 69 genes were up-regulated in the root of NIL-23 (67 in case of stress-tolerant genotype) under the stress, which validates the experimental set up as well as quality/fidelity of data/analysis (Fig. 6, Supplementary Table S3).

Transcriptome data indicated up-regulated expression of some of the RSA genes like glycosyl transferases, expansins, and xyloglucan galactosyl transferases in roots of NIL-23 (Supplementary Table S11). Increased activity of high-affinity $\mathrm{P}$ transporters during P-starvation has been reported to play important role in acquisition of $\mathrm{Pi}$ in plants [74]. Under P-starvation, expression of phosphate transporter 1 (PHT1) group genes for high-affinity $\mathrm{P}$ transporters is induced to increase the ability of roots in acquiring Pi from soils and mobilizing Pi within plants [75]. In the present study, differential expression of 13 OsPHT1s in root/shoot of the contrasting rice genotypes was observed under P-starvation stress (Supplementary Table S4). Expression of OsPHT1;2 (a low-affinity Pitransporter) was reported to increase under P-deficiency stress [41], suggesting its role in transport of $\mathrm{Pi}$ from roots to shoots. Similarly, OsPHT1;6 (a high-affinity Pitransporter) was reported to function in acquiring $\mathrm{Pi}$ from the soil [41]. We observed increased expression of OsPHT1;6 in roots under P-starvation, highest (12.65fold) in NIL-23; thus, our observation corroborate with the finding of $\mathrm{Ai}$ et al. [41] We observed root-specific expression of OsPHT1;3, OsPHT1;5 and OsPHT1;9, which suggest their function in acquisition of Pi from soil. OsPHT1;4, coding for a plasmalemma-localized Pitransporter, was reported to express in root and leaf of rice for acquisition and transport (homeostasis) of Pi [76, 77]. Our findings corroborate with the earlier reports. The up-regulated expression (9.02-fold) of OsPHT1;13 observed in shoot of NIL-23 might be mainly responsible for mobilization of Pi from root to shoot, while it was observed to have major function (10.62-fold up-regulated expression in root under the stress) in acquisition of $\mathrm{P}$ from soil in Pusa-44. Moreover, OsPHT1;12 expression was up-regulated in shoot of both the genotypes compared to that in roots (Supplementary Table S4), which might help mobilizing Pi from root to shoot. Remobilization of Pi within the plant was reported during vegetative and reproductive stage of plant growth [63]. A number of P transporter genes like PHT1;3, PHT1;8, PHT1;10, and PHT1;13 were reported to be induced by nitrate availability [78].

TFs are important regulators of gene expression process, and they have been reported to play key roles in tolerance to various abiotic stresses. Homeobox-domain containing proteins have been reported to bind DNA and regulate transcription of the genes [79]. In the present study, expression of homeobox-domain containing protein and homeobox protein knotted-1 was up-regulated in root of NIL-23 that corroborates with the earlier findings [79]. Likewise, findings of the present study also showed that auxin response factor, AP2 domain containing proteins, GRAS family transcription factor containing protein, histone-like transcription factor, and HSF-type DNA-binding domain containing protein exclusively expressed ( $>2.5$-fold up-regulated) in root of NIL-23 (Supplementary Table S5) which is in agrement with the earlier findings [33, 34]. A P-deficiency-responsive MYB TF was reported earlier to over-express in Arabidopsis under P-starvation [80], which substantiate our finding. Similarly, other P-deficiency-responsive TF families like NAC, AP2, zinc finger, and WRKY [34, 81, 82] were also observed to be differentially expressed in the contrasting rice genotypes under P-starvation (Supplementary Table S5). Though bZIP, MADS, and bHLH TF family genes played important roles in shoot of NIL23, differential expression of MYB family genes played more important roles in root of NIL-23 (Fig. 7). On the other hand, WRKY family genes were observed to play equally important roles in shoot and root for P-starvation tolerance. Various TFs including MYB, ERF/AP2, WRKY, CCAAT-binding, Zinc finger, bHLH, and NAC have been reported to be over-expressed under P-deficiency stress $[32,83]$. A bHLH TF was reported to play role in providing tolerance against P-starvation by improving RSA in rice [82].

Further insights in to the mechanisms involved in P-deficiency stress tolerance came from GO analysis, which deciphered the role of diverse biological/cellular/molecular processes under P-starvation stress tolerance. GO analysis in roots of NIL-23 revealed that a large number of up-regulated genes belong to 229 (more) GO terms, compared to only 175 (less) terms in roots of Pusa-44 (Fig. 8). Among these GO terms, regulation of transcription, protein/amino acid phosphorylation (biological process), and serine/threonine kinase activity, inorganic phosphate transporter activity (molecular function) played important roles in stress tolerance in NIL-23 (Figs. 8, 9). Up-regulated expression of dirigent 
genes in root (At1g64160) and leaf (At2g21100) has been reported in Arabidopsis under P-deficiency [83], which is in agreement with our finding. The Ser/Thr protein phosphatase family proteins modify other proteins by (de)phosphorylation, and regulate cellular functions, signal transduction, and responses to biotic and abiotic stresses [84]. Some of the cellular components like integral membrane component were over-represented (Supplementary Fig. S12), while others like oxygen evolving complex were under-represented (Supplementary Fig. S13) in roots of NIL-23. GO analysis in shoot of NIL-23 indicated exclusive over-representation of some of the biological processes like transcription process, amino acid phosphorylation (Supplementary Fig. S14), and molecular functions like serine/threonine kinase activity (Supplementary Fig. S16), cellular components like phosphofructokinase complex were observed to be exclusively enriched in the shoot of Pusa-44 (Supplementary Fig. S18). Similarly, GO analysis in shoot of NIL-23 indicated that a larger number of down-regulated genes belong to 199 GO terms (Supplementary Figs. S15, S17, S19).

The GO terms associated with carbohydrate, lipid, and nitrogen metabolism were enriched to improve nutritional status, energy supply, and cellular protection, particularly in NIL-23 (Fig. 11). Under P-starvation, cells bypass ATP or Pi-dependent enzymatic reactions of

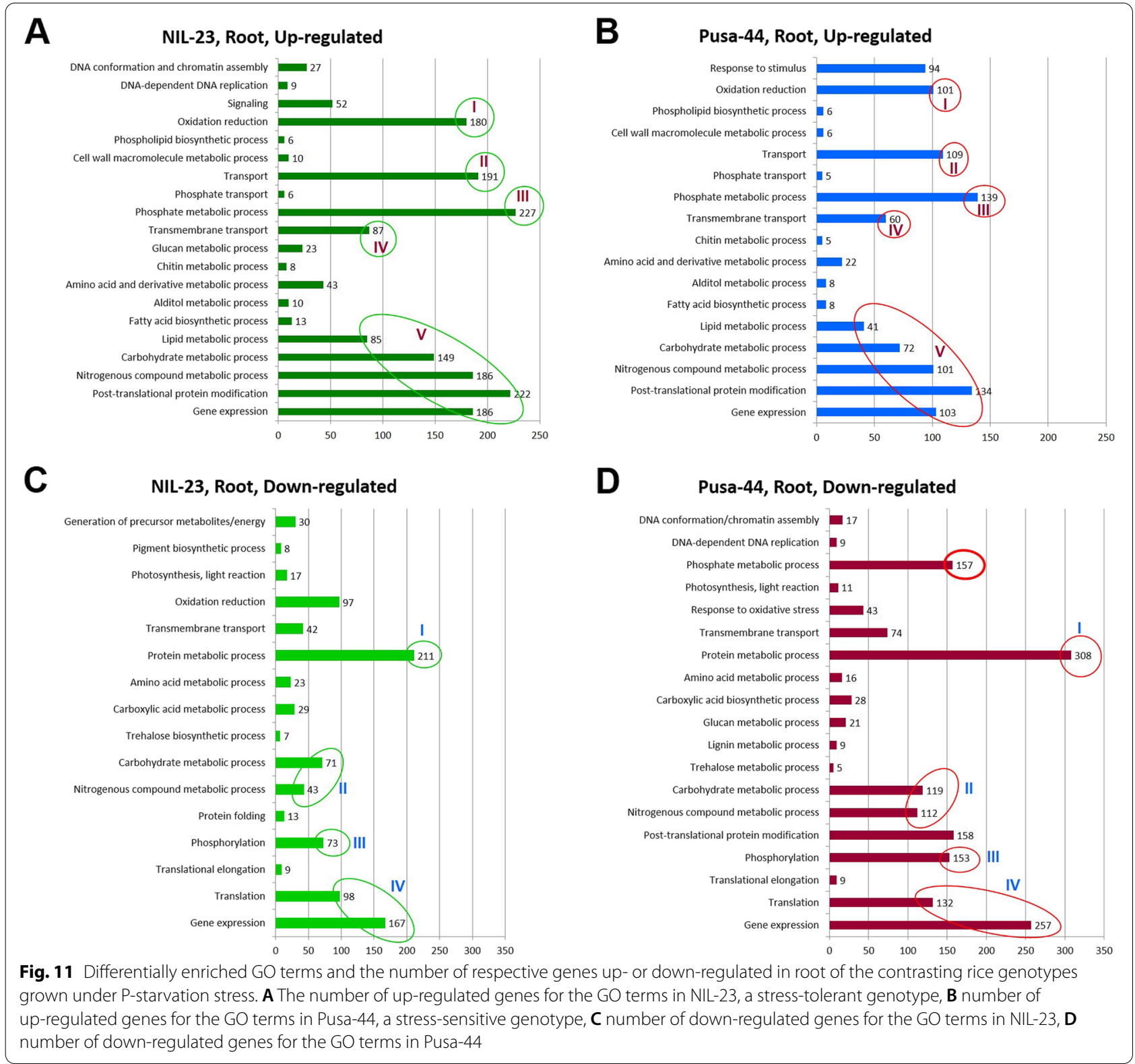


sugar metabolism by using Pi-independent pathways. The genes involved in such glycolytic bypasses were observed to be modulated under the P-starvation stress, particularly in root of NIL-23, which is in agreement with [11]. The first bypass, catalyzed by pyrophosphate-dependent phosphofructokinase (PPi-PFK), converts fructose-6 phosphate to fructose 1,6-bisphosphate without using ATP. PPi-PFK gene (OsO2g48360) was up-regulated in NIL-23, while down-regulated in Pusa-44. The second bypass uses NADP-GAPDH to minimize Pi requirement needed by NAD-GAPDH to form 1,3-bisphosphoglycerate. The NADP-dependent aldehyde dehydrogenase encoding gene (Os12g12590) was up-regulated in root of NIL-23. Third bypass operates downstream in glycolysis by using phosphoenolpyruvate carboxylase (PEPC) and malate dehydrogenase (MDH) to replace pyruvate kinase, which require $\mathrm{Pi}$. The PEPC (Os09g14670) and $\mathrm{MDH}$ encoding genes (Os08g33720) were induced by P-starvation (Supplementary Table S11). The GO terms for phosphorus metabolic process, transmembrane transport, and oxidation-reduction were significantly enriched in roots of NIL-23. Moreover, GO terms/genes for epigenetic regulation of gene expression and post-translational protein modification were differentially represented/ expressed in root/shoot of NIL-23 (Supplementary Table S10).

Cell wall not only provides rigidity to plant, but also controls cell expansion. Therefore, cell often weakens the cell wall structure and maintains turgor/cellular integrity to achieve growth [85]. A subset of genes responsible for cell wall organization and associated activities exhibited up-regulated expression during P-starvation in NIL23 (Supplementary Table S6). Among these, the genes encoding for expansins, LTPL-Protease inhibitor family proteins, and glycine-rich cell wall proteins, etc. were the important players that varied dynamically in roots under P-starvation stress. Expansin and LTPL-protease inhibitor family proteins are known to play important roles in cell wall loosening, wax/cutin deposition in the cell wall of expanding/multiplying cells. Plants deploy signaling mechanisms to sense the developmental and environmental cues and modulate the cell wall structure. Many auxin-responsive genes involved in cell wall biosynthesis and loosening were reported to be up-regulated in siz1 mutant compared to that in the wild-type plants under P-starvation [86]. Our observations on up-regulated expression of auxin-responsive/induced genes support the roles of phytohormones in modulating the cell wall structure in roots of NIL-23 under P-starvation stress (Supplementary Table S7).

The genes involved in membrane-lipid remodeling have been reported to be induced by P-deficiency [87]. Lipid transfer proteins (LTPs), usually localized in extracellular spaces outside the plasma membrane, play important role in deposition/adhesion of wax and lipid barrier polymers. Signaling and tolerance to abiotic/biotic stresses are associated with LTPs [88]. In the present study, several lipid transfer protein (LTP) family genes were dynamically modulated for their expression during P-starvation stress in NIL-23. The genes for membrane lipid remodeling including like phospholipase C, phospholipase D, and glycerophosphodiester phosphodiesterases, were observed to be significantly up-regulated in NIL- 23 compared to that in Pusa-44 under the stress. Pi pool of a cell can also be conserved by substituting phospholipids with sulfolipids and galactolipids in the membrane [89]. Phospholipids being one of the important components of biological membrane, $\mathrm{P}$-deficient plant replaces some of the phospholipids with phosphorus-free galactolipid, sulfoquinovosyl diacylglycerol (SQDG), and digalactosyl diacylglycerol (DGDG) in order to minimize the dependence on $\mathrm{Pi}[11,90,91]$. We observed up-regulated expression of monogalactosyldiacylglycerol (MGDG) synthase (Os08g20420) in root of both the rice genotypes, particularly NIL-23, which is in agreement of the findings of Mehra et al. [11] Thus, Pi deficiency is compromised by increased uptake of sulfur (S) which is clearly indicated by the increased activity of sulfur transporters. Genes involved in membrane-lipid alterations are known to get activated by P-deficiency [87]. Up-regulated expression of $S Q D 1$ and $S Q D 2$ genes, responsible for sulfolipid biosynthesis, was reported to be induced by P-starvation in Arabidopsis and rice $[83,92]$. In the present study also, cell wall reorganization, phospholipid remodeling, and associated activity related genes exhibited up-regulated expression during P-starvation stress in NIL-23. Thus, P-starvation induced lipid remodeling, accumulation of MGDG and DGDG indicate that cellular lipid remobilization is one of the adaptive strategies for P-starvation tolerance in NIL-23, which is in agreement with the earlier reports $[93,94]$.

Modulation in metabolic processes, particularly sugar and lipid metabolism, has been reported to be crucial for adaptation to P-deficiency stress. Increase in sucrose biosynthesis in leaf of Arabidopsis, bean, barley, spinach, and soybean under the stress has been reported [95]. Alteration in diverse metabolic processes related to glucose, pyruvate, sucrose, starch, and chlorophyll under the stress has also been reported $[11,96]$. Decrease in phosphorylated-sugars in leaf and root was reported under the stress because of reduced availability/activity of $\mathrm{Pi} /$ fructokinase/hexokinase [97, 98]. Down-regulated expression of trehalose -6 - phophate synthase was reported under P-deficiency studies [81, 83], which corroborates with our findings. Pi has been also been reported to be involved in regulation of the distribution of fixed carbon 
between starch synthesis (in chloroplast) and transfer of triose-phosphate for sucrose synthesis (in cytoplasm) [99]. P-deficiency was also reported to increase translocation of carbohydrate via phloem to roots to favor root growth for better acquisition of Pi from soil [100]. Thus, more efficient metabolic adjustments in NIL-23, compared to that in Pusa-44, are responsible better survival of NIL-23 under P-starvation stress. Phosphorus homeostasis between shoot and root under the stress has been reported to be maintained by modulating the expression of transporters, phosphatases, RNases, and the enzymes involved in metabolic processes [17, 69, 70, 83].

Photosynthetic process was reported to be inhibited under P-deficiency mainly because of the limiting effect of Pi for ATP synthesis, Rubisco activation, and RuBP regeneration in chloroplasts $[6,101,102]$. P-deficiency supresses Calvin cycle activity by reducing the amount and activity of Rubisco [103] and regeneration of ribulose-1,5-bisphosphate [102]. Moreover, phosphorus plays crucial structural and regulatory roles at the junction of photosynthesis, energy conservation, and carbon metabolism [104]. The balance between anabolic and catabolic carbon metabolism is disrupted under P-deficiency stress. The expression of the genes for photosystem (PS) I, PSII, Rubisco small subunits, Calvin cycle enzymes and chlorophyll A/B-binding proteins have been reported to be repressed by P-deficiency $[105,106]$. At the same time, the genes involved in glycolysis, starch and sucrose synthesis (glucose-6-phosphate dehydrogenase, phosphofructokinase, frucose-1,6-bisphosphate aldolase, phosphoenolpyruvate carboxylase, glyceraldehyde -3 - phosphate dehydrogenase, and sucrose transporters) have been reported to be up-regulated [68, 69], which corroborate with our findings. Such changes are necessary under Pi-deficiency to bypass dependency on the ATP- and Pi-dependent enzymes, and to modulate the metabolic processes required to generate energy and carbon skeleton [107, 108].

Photosynthesis being a most important photochemical sink for energy conversion, P-deficiency mediated inhibition of $\mathrm{CO}_{2}$ assimilation leads to damage to the photosynthetic apparatus by the excessive light/excitation energy. P-deficiency inhibits photophosphorylation process, and limits the availability of ATP/NADPH which affect metabolic processes. We observed $\sim$ sixfold down-regulated expression of a gene (LOC_Os04g16874) for photosystem II reaction center under P-starvation in NIL-23 (Supplementary Table S9), which help protecting the plant from excessive excitation energy. The excessive excitation energy increases the production of reactive oxygen species (ROS); hence, non-photochemical quenching of ROS was reported to increase under P-deficiency in rice [21]. Our findings on differential expression of the genes involved in photosynthesis under stress are in line with that reported by Secco et al. [33]. Modulation in photosynthesis-related genes was observed even in roots of the rice genotypes under the stress, which is in agreement with Li et al. [22] and Wang et al. [36].

Phytohormones like auxin, abscisic acid, cytokinin, and gibberellins are known to play role in root development [109]. Auxin plays role in the development of lateral roots and thus helps acquisition of phosphorus. In Arabidopsis, cell cycle in lateral roots is regulated by auxin, and P-deficiency affects expression of the cell cycle genes like $C D K A, E 2 F a, D p-E 2 F$ and $C y C D 3$. Thus, modification in root architecture is associated with P-starvation through phytohormones. A linkage between auxin transporters PIN7 and APSR1 was reported because absence of ASPR1 decreased expression of PIN7 under P-starvation [110]. In addition, P-starvation reduces gibberellic acid (GA) content leading to increased DELLA proteins [111], which resulted in increased length of root hairs in NIL23 under the stress.

Chromatin architecture is a key determinant of gene expression in eukaryotes. Certain chromatin-related components have been reported to play roles in root hair growth during P-deficiency stress in Arabidopsis. A histone deacetylase (HDA19) was reported to get induced and increased root hair growth under P-deficiency in Arabidopsis [112]. Zahraeifard et al. [113] reported alteration in genome-wide H2A.Z distribution due to P-starvation in rice. Zhang et al. [114] suggested the role of nucleosome remodelers in modulating nucleosome occupancy and differential gene expression under P-deficiency stress. A possible modulation in chromatin architecture can be expected under the stress due to the observed differential expression of jmjC domain containing protein, core histone $\mathrm{H} 2 \mathrm{~A} / \mathrm{H} 2 \mathrm{~B} / \mathrm{H} 3 / \mathrm{H} 4$ domain containing proteins, $\mathrm{C}-5$ cytosine-specific DNA methylase, and methyl-CpG binding domain containing protein. An extensive remodeling of global DNA methylation under P-deficiency stress in Arabidopsis was reported by YongVillalobos et al. [115] Hypo- and hyper-methylation of DNA in the vicinity of TF binding sites under P-starvation in Arabidopsis was reported by Yong-Villalobos et al. [116] Our observation on DNA methylase to be up-regulated and methyl-CpG binding domain containing protein to be down-regulated in the stress tolerant genotype under the stress (Supplementary Table S10) corroborate with the above findings. Similarly, differential expression of P-starvation-responsive proteins like histone chaperone (in our case hsp20) and nucleosome assembly protein were reported in Arabidopsis under P-starvation stress [91].

Several QTLs have been detected on different chromosomes in rice for P uptake, among which Pup1 is the 
major one. Although ectopic expression of PSTOL1 in P-deficiency sensitive rice cultivar was found to enhance early root growth enabling plants to acquire more phosphorus, as well as other nutrients, and increase grain yield in P-deficient soil [29], the role of Pup1 is not clear. We observed up-regulated expression of fatty acid oxygenases, dirigent proteins, aspartic proteinases, and protein kinases genes, particularly in root of NIL-23 under P-starvation stress (Supplementary Table S11). Since the genes on Pup1 QTL do not code for a known P uptakerelated protein, the mode of action of $P u p 1$ is still unclear. However, OsPupK20, OsPupK29, and OsPupK46 (protein kinase genes) have been reported to be the candidate genes associated with root-specific functions $[34,117]$.

\section{Conclusions}

As $\mathrm{P}$ is the second most important macronutrient for the growth and development of plants, it is unfortunate that many aspects of $\mathrm{P}$ uptake, transport and useefficiency in plants are not yet thoroughly understood. Some of the studies provide insights into P distribution, functional characteristics of the transporters, and differential expression of the genes involved in P-deficiency tolerance. The present study reveals significant roles of the transporters (OsPHT1;6, OSPHT1;10), signalling molecules like jasmonate/auxin-induced proteins (LOC_ Os04g22900, LOC_Os01g36580), auxin-responsive protein (LOC_Os05g48270), LTPL-protease inhibitor family proteins (LOC_Os10g40430, LOC_Os10g40480), glycerophosphoryl diester phosphodiesterase family protein (LOC_Os03g40670), phosphatases (LOC_ Os01g52230, LOC_Os08g17784, LOC_Os11g38050, LOC_Os07g01540, LOC_Os05g02310), transcription factors like homeobox domain containing protein (LOC Os03g51690), MYB family TFs (LOC_Os03g62100, LOC_Os02g22020) and AP2 domain containing proteins (LOC_Os08g36920, LOC_Os10g11580), core histone domain containing protein (LOC_Os10g28230), and glycine-rich cell wall structural proteins (LOC_Os10g31530, LOC_Os10g31540) in providing P-starvation stress tolerance in rice. Better P-use efficiency and lower Pi demand of NIL-23, because of introgression of the Pup1 QTL in the Pusa-44 genetic background, are responsible for better growth and survival of NIL-23 plants until vegetative stage even under P-starvation stress. The present study offers plentiful molecular information/candidate mechanisms for functional dissection of P-deficiency stress tolerance in rice, which might further help understanding the regulatory network by integrating the biochemical, physiological, genetic, and molecular mechanisms to enhance P-use efficiency in crop plants to cope with the limiting availability of $\mathrm{P}$ in the soil (Fig. 12), particularly because of the changing environmental conditions. Thus, the mechanisms like modulation in root system architecture, $\mathrm{P}$ acquisition, internal $\mathrm{Pi}$ remobilization, energy conservation might help improving P-use efficiency in rice through genetic and epigenetic approaches to improve productivity of modern cultivars in P-deficient soils.

\section{Methods \\ Plant materials, growth conditions and P-starvation stress imposition}

Two contrasting rice genotypes [Pusa-44 (P-deficiency sensitive) and NIL-23 (P-deficiency tolerant) received from the Division of Genetics, ICAR-Indian Agricultural Research Institute, New Delhi] were used in the present study. Mature seeds of the contrasting rice genotype [along with the original Pup1 QTL donor (Kasalath), received from the Division of Genetics, ICAR-Indian Agricultural Research Institute, New Delhi] were used to raise seedlings/plants hydroponically in P-sufficient (16 ppm Pi), P-deficient (1 or $4 \mathrm{ppm}$ ) or P-starvation (0 ppm Pi) condition. More details of the rice genotypes (Supplementary Figs. S20, S21), growth conditions (Supplementary Fig. S22) and P-starvation stress have been provided in Supplementary Methods. Shoot and root tissues collected from 45-day-old plants (in vegetative/tillering stage) were used for various analyses.

\section{Estimation of APase activity, root - shoot biomass ratio, chlorophyll and phosphorus contents}

Acid phosphatase (APase) activity in shoot and root tissues, secreted APase from roots, and Root-shoot biomass ratio were estimated/calculated. Total chlorophyll content in leaf was estimated using dimethyl sulfoxide method. Total phosphorus content in shoot and root tissues was determined using Vanadate-molybdate method. The detailed procedure of the above-mentioned analyses is provided as Supplementary Methods.

\section{RNA isolation, library preparation, and data analyses}

To analyze the effects of P-starvation stress on gene expression in shoot and root of the contrasting rice genotypes, total of 16 libraries were prepared, and got sequenced commercially on Illumina platform using PE $2 \times 150$ bp chemistry. The raw sequence data was submitted to NCBI Sequence Read Archive (SRA) database under the BioProject ID PRJNA667189. Reference-based mapping of the RNA-seq data was performed using the rice reference genome (TIGR v7) with the help of Hisat2 and Stringtie package. The number of mapped clean reads for each gene was counted and normalized into the reads per kilo base per million (RPKM) value. Differentially expressed genes (DEGs) were annotated with gene ontology terms and key pathways via functional classification 


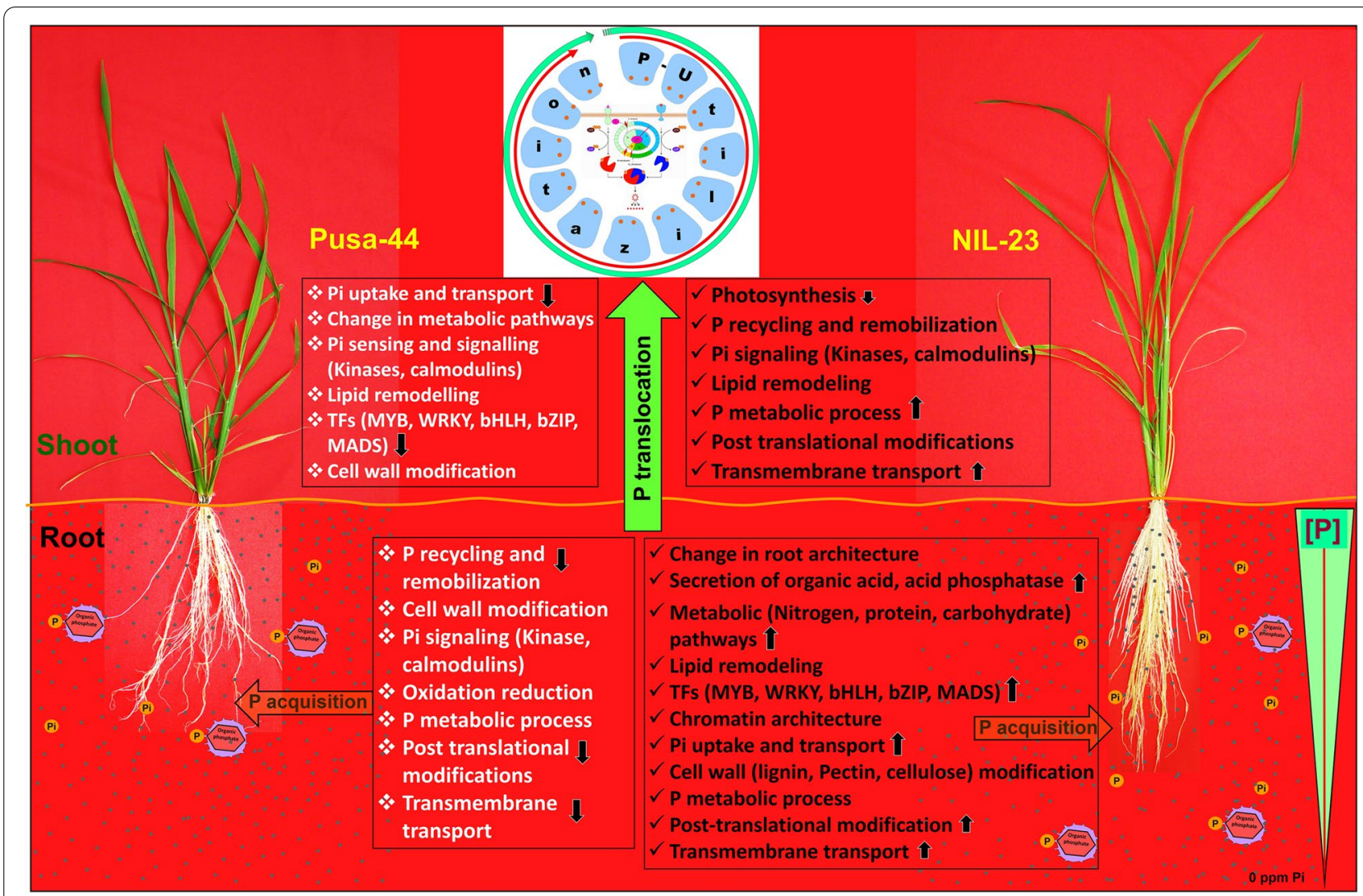

Fig. 12 Diagrammatic representation of the mechanisms involved in P-deficiency stress tolerance in rice (Pusa-44, stress sensitive, and NIL-23, stress tolerant). The upward arrow $(\uparrow)$ indicates higher/supportive role of the mechanism, while the downward arrow $(\downarrow)$ indicates down-regulation of the process in under the stress

and Kyoto Encyclopedia of Gene and Genomes pathway mapping, respectively. DEGs were compared between control and treatment, as well as between the rice genotypes. Genes with $\log _{2}$ fold-changes $>1$ (i.e., foldchange $>2)$ and $p<0.05$ were considered differentially expressed. Detail procedures of RNA isolation, library preparation, and data analysis, etc. are provided in the Supplementary Methods.

\section{Validation of differentially expressed genes by RT-qPCR}

To confirm the results of RNA-seq, seven DEGs (four up-regulated and three down-regulated) were selected randomly for quantitative (RT-qPCR) analysis following the MIQE guidelines. The data was analyzed through melt-curve to check specificity of the PCR amplification. The relative gene expression was determined by the $2^{-}$ $\Delta \Delta \mathrm{Ct}$ method. Actin and tubulin genes were used as reference/housekeeping genes. More details of the RT-qPCR validation are provided in the Supplementary Methods, and the primers used are listed in Supplementary Table S12.
This is confirmed that all the materials and methods used for the present research work complied with relevant institutional, national, and international guidelines and legislation.

\section{Abbreviations}

APase: Acid phosphatase; DEGs: Differentially expressed genes; FC: Fold change; FDR: False discovery rate; GA: Gibberellic acid; DGDG: Digalactosyl diacylglycerol; GO: Gene ontology; LTP: Lipid transfer protein; MGDG: Monogalactosyl diacylglycerol; MGDGS: Monogalactosyl diacylglycerol synthase; NIL: Near-isogenic line; PHT1: Phosphorus transporter 1; P: Phosphorus; Pi: Inorganic phosphorus; ppm: Part per million; PS: Photosystem; Pup1: Phosphorus uptake 1; QTL: Quantitative trait loci; RSA: Root system architecture; RuBP: Ribulose-1,5-bisphosphate; SQDG: Sulfoquinovosyl diacylglycerol; TF: Transcription factor.

\section{Supplementary Information}

The online version contains supplementary material available at https://doi. org/10.1186/s12870-021-03015-4.

Additional file 1: Supplementary Tables S1 to S2. Comparative analysis of root-shoot biomass ratio in the contrasting rice genotypes along with the donor parent, Effect of the root-secreted acid phosphatase on $\mathrm{pH}$ of the hydroponic medium. 
Additional file 2: Supplementary Tables S3 to S11. Expression level of the P-starvation-inducible genes, 13 Phosphorus transporters during P-starvation stress, Differentially expressed Transcription Factors, Cell wall associated genes in root of the contrasting rice genotypes, Phytohormone encoding genes, Genes associated with carbohydrate and lipid metabolism, Photosynthesis related genes, Genes associated with epigenetic regulation of gene expression, Master data-sheet of the genes detected for their expression in the contrasting rice genotypes under P-starvation stress.

Additional file 3: Supplementary Table S12. List of the primers used for RT-qPCR validation of the randomly selected differentially expressed genes (DEGs) in the contrasting rice (Pusa-44, P-deficiency stress sensitive; NIL-23, P-deficiency tolerant) genotypes.

Additional file 4: Supplementary Methods. Detailed method for the experiments performed.

Additional files 5: Supplementary Figures S1 to S22.

\section{Acknowledgements}

SK acknowledges funding from Extramural Research Grant of the Division of Crop Sciences, Indian Council of Agricultural Research, New Delhi, India.

\section{Authors' contributions}

SK and TM conceived and designed the experiments; SK supervised the experiments, data collection, and analyses; Pallavi and CC performed morphological, physiological and biochemical experiments; KS performed molecular analyses; KKV developed Near Isogenic Lines, evaluated them and provided the best performers for experimentation; $S K^{2}$ performed bioinformatics analysis of the data. SK, and SK' , prepared the manuscript, SK, SK², KKV, and TM revised the manuscript, and all the authors approved the final draft.

\section{Funding}

The research was carried out with a financial support from Extramural Research Grant [18(3)/2018-O\&P] from the Indian Council of Agricultural Research, Govt. of India, New Delhi, India.

\section{Availability of data and materials}

The morphophysiological and biochemical data generated/analyzed during this study are included in the Supplementary files. RNA-sequencing (transcriptome) raw reads data are available at NCBI Sequence Read Archive (SRA) database (https://www.ncbi.nlm.nih.gov/sra) under the BioProject ID: PRJNA667189.

\section{Declarations}

\section{Ethics approval and consent to participate}

Not applicable.

\section{Consent for publication}

Not applicable.

\section{Competing interests}

The authors declare that they have no competing interests. $S K^{2}$ is employed at the Decode Genomics Private Limited, New Delhi, and the work was conducted in the absence of any commercial or financial relationships that could be construed as a potential conflict of interest.

\section{Author details}

'Division of Biochemistry, ICAR-Indian Agricultural Research Institute, New Delhi 110012, India. ${ }^{2}$ Present Address: J.N.L. College, Patliputra University, Patna, Bihar, India. ${ }^{3}$ Decode Genomics Private Limited, New Delhi, India. ${ }^{4}$ Division of Genetics, ICAR-Indian Agricultural Research Institute, New Delhi, India. ${ }^{5}$ Indian Council of Agricultural Research, New Delhi, India.

Received: 3 February 2021 Accepted: 5 May 2021

Published online: 21 June 2021

\section{References}

1. Secco D, Wang C, Shou H, Schultz MD, Chiarenza S, Naussaume L, et al. Stress induced gene expression drives transient DNA methylation changes at adjacent repetitive elements. eLife. 2015; doi:https://doi. org/10.7554/eLife.09343.001

2. Poirier Y, Bucher M. Phosphate transport and homeostasis in Arabidopsis. The Arabidopsis Book 1. 2002:e0024. doi:https://doi.org/10.1199/tab. 0024

3. Schröder JJ, Smit AL, Cordell D, Rosemarin A. Improved phosphorus use efficiency in agriculture: a key requirement for its sustainable use. Chemosphere. 2011;84:822-31.

4. Wu P, Shou H, Xu G, Lian X. Improvement of phosphorus efficiency in rice on the basis of understanding phosphate signaling and homeostasis. Curr Opin Plant Biol. 2013;16:205-12.

5. Aluwihare YC, Ishan M, Chamikara MDM, Weebadde CK, Sirisena DN, Samarasinghe WLG, Sooriyapathirana SDSS. Characterization and selection of phosphorus deficiency tolerant rice genotypes in Sri Lanka. Rice Sci. 2016;23:184-95.

6. Wissuwa M, Gamat G, Ismail AM. Is root growth under phosphorus deficiency affected by source or sink limitations? J Exp Bot. 2005;56:1943-50.

7. Sanyal SK, Dwivedi BS, Singh VK, Majumdar K, Datta SC, Pattanayak SK. Annapurna K Phosphorus in relation to dominant cropping sequences in India: chemistry, fertility relations and management options. Curr Sci. 2015;108:1262-70.

8. Li ZJ, Xie Y, Dai AY, Liu LF, Li ZC. Root and shoot traits responses to phosphorus deficiency and QTL analysis at seedling stage using introgression lines of rice. J Genet Genomics. 2009;36:173-83.

9. Li Y, Gu M, Zhang , Zhang J, Fan H, Li P, Li Z, Xu G. Engineering a sensitive visual-tracking reporter system for, real-time monitoring phosphorus deficiency in tobacco. Plant Biotechnol J. 2014;12:674-84.

10. Elser JJ. Phosphorus: a limiting nutrient for humanity? Curr Opin Biotechnol. 2012;23:833-8. https://doi.org/10.1016/j.copbio.2012.03.001.

11. Mehra P, Pandey BK, Giri J. Comparative morphophysiological analyses and molecular profiling reveal Pi-efficient strategies of a traditional rice genotype. Front Plant Sci. 2016;6:1184. https://doi.org/10.3389/fpls. 2015.01184

12. Wang YS, Jensen LS, Magid J. Differential responses of root and root hair traits of spring wheat genotypes to phosphorus deficiency in solution culture. Plant Soil Environ. 2016:62:540-6.

13. Schachtman DP, Reid RJ, Ayling SM. Phosphorus uptake by plants: from soil to cell. Plant Physiol. 1998;116:447-53.

14. Weisskopf L, Fromin N, Tomasi N, Aragno M, Martinoia E. Secretion activity of white lupin's cluster roots influences bacterial abundance, function and community structure. Plant Soil. 2005;268:181-94. https:// doi.org/10.1007/s11104-004-0264-X.

15. Holz M, Zarebanadkouki M, Kuzyakov Y, Pausch J, Carminati A. Root hairs increase rhizosphere extension and carbon input to soil. Ann Bot. 2018;121:61-9. https://doi.org/10.1093/aob/mcx127.

16. Tian J, Wang C, Zhang Q, He X, Whelan J, Shou H. Over expression of OSPAP10, a root-associated acidphosphatase, increased extracellular organic phosphorus utilization in rice. J Integr Plant Biol. 2012;54:631-9.

17. Gho Y-S, Choi H, Moon S, Song MY, Park HE, Kim D-H, Ha S-H, Jung K-H. Phosphate-starvation-inducible S-like RNase genes in rice are involved in phosphate source recycling by RNA decay. Front Plant Sci. 2020;11: 585561. https://doi.org/10.3389/fpls.2020.585561.

18. Gu M, Zhang J, Li H, Meng D, Li R, Dai X, et al. Maintenance of phosphate homeostasis and root development are coordinately regulated by MYB1, an R2R3-type MYB transcription factor in rice. J Exp Bot. 2017:68:3603-15.

19. Chang MX, Gu M, Xia Y, Dai X, Dai C, Zhang J, et al. OsPHT13 mediates uptake translocation and remobilization of phosphate under extremely low phosphate regimes. Plant Physiol. 2019;179:656-70.

20. El Mazlouzi M, Morel C, Robert T, Yan B, Mollier A. Phosphorus uptake and partitioning in two durum wheat cultivars with contrasting biomass allocation as affected by different $P$ supply during grain filling. Plant Soil. 2020;449:179-92. https://doi.org/10.1007/ s11104-020-04444-0.

21. Xu HX, Weng $X Y$, Yang $Y$. Effect of phosphorus deficiency on the photosynthetic characteristics of rice plants. Russian J Plant Physiol. 2007:54:741-8. 
22. Li L, Liu C, Lian X. Gene expression profiles in rice roots under low phosphorus stress. Plant Mol Biol. 2010;72:423-32.

23. Xing D, Wu Y. Effect of phosphorus deficiency on photosynthetic inorganic carbon assimilation of three climber plant species. Bot Stud. 2014;55:60.

24. Sun $Y, M u C$, Chen $Y$, Kong $X, X u Y$, Zheng $H$, et al. Comparative transcript profiling of maize inbreds in response to long-term phosphorus deficiency stress. Plant Physiol Biochem. 2016;109:467-81.

25. Zhang J, Jiang F, Shen Y, Zhan Q, Bai B, Chen W, Chi Y. Transcriptome analysis reveals candidate genes related to phosphorus starvation tolerance in sorghum. BMC Plant Biol. 2019;19:306. https://doi.org/10. 1186/s12870-019-1914-8

26. Khush GS. What it will take to feed 5.0 billion rice consumers in 2030. Plant Mol Biol. 2005;59:1-6. doi:https://doi.org/10.1007/ s11103-005-2159-5

27. Wissuwa M, Yano M, Ae N. Mapping of QTLs for phosphorus deficiency tolerance in rice (Oryza sativa L.). Theor Appl Genet. 1998:97:777-783.

28. Hufnagel B, de Sousa SM, Assis L, et al. Duplicate and conquer: multiple homologs of phosphorus-starvation tolerance 1 enhance phosphorus acquisition and sorghum performance on low-phosphorus soils. Plant Physiol. 2014;166:659-767.

29. Gamuyao R, Chin HJ, Tanaka JP, Pesaresi P, Catausan S, Dalid C, et al. The protein kinase Psto/1 from traditional rice confers tolerance of phosphorous deficiency. Nature. 2012;488:535-41. https://doi.org/10. 1038/nature11346

30. Neelam K, Thakur S, Neha, Yadav IS, Kumar K, Dhaliwal, SS, Singh K Novel alleles of phosphorus-starvation tolerance 1 gene (PSTOL1) from Oryza rufipogon confers high phosphorus uptake efficiency. Front Plant Sci. 2017;8:509. doi:https://doi.org/10.3389/fpls.2017. 00509

31. Heuer S, Lu X, Chin JH, Tanaka JP, Kanamori H, Matsumoto T, et al. Comparative sequence analyses of the major quantitative trait locus phosphorus uptake 1 (Pup 1) reveal a complex genetic structure. Plant Biotechnol J. 2009;7:456-7.

32. Hammond JP, Bennett MJ, Bowen HC, Broadley MR, Eastwood DC, et al. Changes in gene expression in Arabidopsis shoots during phosphate starvation and the potential for developing smart plants. Plant Physiol. 2003;132:578-96.

33. Secco $D$, Jabnoune $M$, Walker $H$, Shou $H$, Wu P, Poirier $Y$, Whelan J. Spatio-temporal transcript profiling of rice roots and shoots in response to phosphate starvation and recovery. Plant Cell. 2013;25:4285-304.

34. Deng Q-W, Luo X-D, Chen Y-L, Zhou Y, Zhang FT, Hu B-L, Xie J-K. Transcriptome analysis of phosphorus stress responsiveness in the seedlings of Dongxiang wild rice (Oryza rufipogon Griff.). Biol Res. 2018;51:7. doi:https://doi.org/10.1186/s40659-018-0155-X

35. Ren P, Meng Y, Li B, Ma X, Si E, Lai Y, et al. Molecular mechanisms of acclimatization to phosphorus starvation and recovery underlying full-length transcriptome profiling in barley (Hordeum vulgare L.). Front Plant Sci. 2018;9:500. doi:https://doi.org/10.3389/fpls.2018.00500

36. Wang J, Qin Q, Pan J, Sun L, Sun Y, Xue Y, Song K. Transcriptome analysis in roots and leaves of wheat seedlings in response to lowphosphorus stress. Sci Rep. 2019:9:19802. https://doi.org/10.1038/ s41598-019-56451-6.

37. Foroozani M, Zahraeifard S, Oh D-H, Wang G, Dassanayake M, Smith AP. Low-phosphate chromatin dynamics predict a cell wall remodeling network in rice shoots. Plant Physiol. 2020;182:1494-509.

38. Mudge SR, Rae AL, Diatloff E, Smith FW. Expression analysis suggests novel roles for members of the PHT1 family of phosphate transporters in Arabidopsis. Plant J. 2002;31:341-53.

39. Goff SA, Ricke D, Lan TH, et al. A draft sequence of the rice genome (Oryza sativa L. ssp japonica). Science. 2020;296:92-100.

40. Paszkowski U, Kroken S, Roux C, Briggs SP. Rice phosphate transporters include an evolutionarily divergent gene specifically activated in arbuscular mycorrhizal symbiosis. Proc Natl Acad Sci USA. 2002;99:13324-9.

41. Ai P, Sun S, Zhao J, Fan X, Xin X, Guo Q, et al. Two rice phosphate transporters, OsPht 1;2 and OsPht1;6, have different functions and kinetic properties in uptake and translocation. Plant J. 2009;57:798-809.

42. Sun S, Gu M, Cao Y, Huang X, Zhang X, Ai P, et al. A constitutive expressed phosphate transporter OsPht1;1 modulates phosphate uptake and translocation in phosphate-replete rice. Plant Physiol. 2012;159:1571-81.

43. Jia H, Zhang S, Wang L, Yang Y, Zhang H, Cui H, et al. OsPht18 a phosphate transporter is involved in auxin and phosphate starvation response in rice. J Exp Bot. 2017;68:5057-68.

44. Yamaji N, Takemoto Y, Miyaji T, Mitani-Ueno N, Yoshida KT, Ma JF Reducing phosphorus accumulation in rice grains with an impaired transporter in the node. Nature. 2017;5(541):92-5.

45. Wang Z, Hu H, Huang H, Duan K, Wu Z, Wu P. Regulation of OsSPX 1 and OsSPX3 on expression of OsSPX domain genes and Pi-starvation signaling in rice. J Integr Plant Biol. 2009;51:663-74.

46. Kobayashi K, Awai K, Nakamura M, Nagatani A, Masuda T, Ohta H. TypeB monogalactosyl diacylglycerol synthases are involved in phosphate starvation-induced lipid remodeling, and are crucial for low-phosphate adaptation. Plant J. 2009;57:322-31.

47. Banerjee A, Roychoudhury A. WRKY proteins: signaling and regulation of expression during abiotic stress responses. Sci World J. 2015;807560.

48. Phukan UJ, Jeena GS, Shukla RK. WRKY transcription factors: Molecular regulation and stress responses in plants. Front Plant Sci. 2016;7:760.

49. Wang $H, X u$ Q, Kong YH, Chen $Y$, Duan JY, Chen YF, et al. Arabidopsis WRKY45 transcription factor activates PHOSPHATE TRANSPORTER1;1 expression in response to phosphate starvation. Plant Physiol. 2014;164:2020-9. https://doi.org/10.1104/pp.113.235077.

50. Baek D, Chun HJ, Yun DJ, Kim MC. Cross-talk between phosphate starvation and other environmental stress signaling pathways in plants. Mol Cell. 2017:40:697-770.

51. Chiou TJ, Lin SI. Signaling network in sensing phosphate availability in plants. Annu Rev Plant Biol. 2011;62:185-206.

52. Baek D, Kim MC, Chun HJ, Kang S, Park HC, Shin G, et al. Regulation of miR399f transcription by AtMYB2 affects phosphate starvation responses in Arabidopsis. Plant Physiol. 2013;161:362-73.

53. Chien PS, Chiang CB, Wang Z, Chiou TJ. Micro RNA-mediated signaling and regulation of nutrient transport and utilization. Curr Opin Plant Biol. 2017;39:73-9. https://doi.org/10.1016/j.pbi.2017.06.007.

54. Ribot C, Wang Y, Poirier Y. Expression analyzes of three members of the AtPHO1 family reveal differential interactions between signaling pathways involved in phosphate deficiency and the responses to auxin, cytokinin and abscisic acid. Planta. 2008;227:1025-36.

55. Trull MC, Guiltinan MJ, Lynch JP, Deikman J. The responses of wild-type and ABA mutant Arabidopsis thaiiana plants to phosphorus starvation. Plant Cell Environ. 1997;20:85-92.

56. Jiang C, Gao X, Liao L, Harberd NP, Fu X. Phosphate starvation root architecture and anthocyanin accumulation responses are modulated by the gibberellin-DELLA signaling pathway in Arabidopsis. Plant Physiol. 2007;145:1460-70.

57. Khan GA, Vogiatzaki E, Glauser G, Poirier Y. Phosphate deficiency induces the jasmonate pathway and enhances resistance to insect herbivory. Plant Physiol. 2016;171:632-44.

58. Wei R, Wang X, Zhang W, Shen J, Zhang H, Gao Y, et al. The improved phosphorus utilization and reduced phosphorus consumption of ppkexpressing transgenic rice. Field Crops Res. 2020;248:107715.

59. Yuan H, Liu D. Signaling components involved in plant responses to phosphate starvation. J Integr Plant Biol. 2008;50:849-59.

60. Sharma S, Borah P, Meena MK, Bindraban P, Pandey R. Evaluation of genotypic variation for growth of rice seedlings under optimized hydroponics medium. Indian J Genet. 2018;78:292-301.

61. Nguyen V, Stangoulis J. Variation in root system architecture and morphology of two wheat genotypes is a predictor of their tolerance to phosphorus deficiency. Acta Physiol Plant. 2019;41:109.

62. Filstrup CT, Downing JA. Relationship of chlorophyll to phosphorus and nitrogen in nutrient-rich lakes. Inland Waters. 2017;7:385-400. https:// doi.org/10.1080/20442041.2017.1375176.

63. Carstensen A, Szameitat AE, Frydenvang J, Husted S. Chlorophyll a fluorescence analysis can detect phosphorus deficiency under field conditions and is an effective tool to prevent grain yield reductions in spring barley (Hordeum vulgare L.). Plant Soil 2019:434:79-91.

64. Vance $\mathrm{CP}$, Uhde-Stone $\mathrm{C}$, Allan DL. Phosphorus acquisition and use: critical adaptations by plants for securing a nonrenewable resource. New Phytol. 2003;157:423-47. 
65. Péret B, Clement M, Nussaume L, Desnos T. Root developmental adaptation to phosphate starvation: better safe than sorry. Trends Plant Sci. 2011;16:442-50. https://doi.org/10.1016/j.tplants.

66. Fohse D, Claasse N, Jungk A. Phosphorus deficiency of plants I. External and internal $P$ requirement and $P$ uptake efficiency of different plant species. Plant Soil. 1988;110:101-109.

67. Yugandhar P, Nallamothu V, Panigrahy M, Tipireddy S, Bhadana VP, Voleti SR, et al. Nagina 22 mutants tolerant or sensitive to low $P$ in field show contrasting response to double $\mathrm{P}$ in hydroponics and pots. Arch Agron Soil Sci. 2018;64:1975-87.

68. Uhde-Stone C, Zinn KE, Ramirez-Yáñez M, Li A, Vance CP, Allan DL. Nylon filter arrays reveal differential gene expression in proteoid roots of white lupin in response to phosphorus deficiency. Plant Physiol. 2003;131:1064-79.

69. Morcuende R, Bari R, Gibon Y, Zheng W, Pant BD, Bläsing O, et al. Genome-wide reprogramming of metabolism and regulatory networks of Arabidopsis in response to phosphorus. Plant Cell Environ. 2007:30:85-112.

70. Calderon-Vazquez C, Ibarra-Laclette E, Caballero-Perez J. Herrera-Estrella $L$ Transcript profiling of Zea mays roots reveals gene responses to phosphate deficiency at the plant- and species-specific levels. J Exp Bot. 2008:59:2479-524.

71. Liang C, Wang J, Zhao J, Tian J, Liao H. Control of phosphate homeostasis through gene regulation in crops. Curr Opin Plant Biol. 2014;21:59-66.

72. Xu L, Jin L, Long L, Liu L, He X, Gao W, Zhu L, Zhang X. Overexpression of GbWRKY 1 positively regulates the Pi starvation response by alteration of auxin sensitivity in Arabidopsis. Plant Cell Rep. 2012;31:2177-88.

73. York LM, Nord EA, Lynch JP. Integration of root phenes for soil resource acquisition. Front Plant Sci. 2013;4:355. https://doi.org/10.3389/fpls. 2013.00355.

74. Shin H, Shin HS, Dewbre GR, Harrison MJ. Phosphate transport in Arabidopsis: Pht1;1 and Pht1;4 play a major role in phosphate acquisition from both low- and high-phosphate environments. Plant J. 2004:39:629-42.

75. Mudge SR, Smith FW, Richardson AE. Root-specific and phosphateregulated expression of phytase under the control of a phosphate transporter promoter enables Arabidopsis to grow on phytate as a sole P source. Plant Sci. 2003;165:871-8.

76. Ye Y, Yuan J, Chang X, Yang M, Zhang L, Lu K, et al. The phosphate transporter gene OsPht $1 ; 4$ is involved in phosphate homeostasis in rice. PLoS ONE. 2015;10: e0126186. https://doi.org/10.1371/journal.pone. 0126186.

77. Zhang F, Sun Y, Pei W, Jain A, Sun R, Cao Y, et al. Involvement of OsPht 1;4 in phosphate acquisition and mobilization facilitates embryo development in rice. Plant J. 2015:82:556-69.

78. Hu B, Jiang Z, Wang W, Qiu Y, Zhang Z, Liu Y, et al. Nitrate-NRT1.1BSPX4 cascade integrates nitrogen and phosphorus signalling networks in plants. Nat Plant. 2019;doi:https://doi.org/10.1038/ s41477-019-0384-1

79. Bürglin TR, Afolter M. Homeodomain proteins: an update. Chromosoma. 2016;125:497.

80. Devaiah BN, Madhuvanthi R, Karthikeyan AS, Raghothama KG. Phosphate starvation responses and gibberellic acid biosynthesis are regulated by the MYB62 transcription factor in Arabidopsis. Mol Plant. 2009;2:43-58

81. Nilsson L, Müller R, Nielsen TH. Dissecting the plant transcriptome and the regulatory responses to phosphate deprivation. Physiol Plant. 2010;139:129-43. https://doi.org/10.1111/j.1399-3054.2010.01356.x.

82. Dia $X$, Wang $Y$, Zhang WH. OsWRKY74, a WRKY transcription factor, modulates tolerance to phosphate starvation in rice. J Exp Bot. 2016:67:947-60.

83. Misson J, Raghothama KG, Jain A, Jouhet J, Block MA, Bligny R, et al. A genome-wide transcriptional analysis using Arabidopsis thaliana Affymetrix gene chips determined plant responses to phosphate deprivation. Proc Natl Acad Sci USA. 2005;102:11934-9.

84. Chen YL, Hung MH, Chu PY, Chao TI, Tsai MH, Chen LJ, et al. Protein phosphatase 5 promotes hepatocarcinogenesis through interaction with AMP-activated protein kinase. Biochem Pharmacol. 2017;138:49-60
85. Voxeur A, Höfte H. Cell wall integrity signaling in plants: "To grow or not to grow that's the question." Glycobiology. 2016;26:950-60.

86. Miura K, Lee J, Gong Q, Ma S, Jin JD, Yoo CY, et al. SIZ1 regulation of phosphate starvation-induced root architecture remodeling involves the control of auxin accumulation. Plant Physiol. 2011;155:1000-12.

87. Nakamura Y, Koizumi R, Shui G, Shimojima M, Wenk MR, Ito T, Ohta H. Arabidopsis lipins mediate eukaryotic pathway of lipid metabolism and cope critically with phosphate starvation. Proc Natl Acad Sci USA. 2009;106:20978-83.

88. Edqvist J, Blomqvist K, Nieuwland J, Salminen TA. Plant lipid transfer proteins: are we finally closing in on the roles of these enigmatic proteins. J Lipid Res. 2018;59:1374-82.

89. Okazaki Y, Otsuki H, Narisawa T, Kobayashi M, Sawai S, Kamide Y, et al. A new class of plant lipid is essential for protection against phosphorus depletion. Nat Commun. 2013;4:1510. https://doi.org/10.1038/ncomm s2512.

90. Gaude N, Nakamura Y, Scheible WR, Ohta H, Dörmann P. Phospholipase C5 (NPC5) is involved in galactolipid accumulation during phosphate limitation in leaves of Arabidopsis. Plant J. 2008;56:28-39.

91. Bhardwaj D, Medici A, Gojon A, Lacombe B, Tuteja N. A new insight into root responses to external cues: Paradigm shift in nutrient sensing. Plant Signal Behav. 2015;10:e104979.

92. Wang XM, Yi KK, Tao Y, Wang F, Wu ZC, Jiang DA, et al. Cytokinin represses phosphate-starvation response through increasing of intracellular phosphate level. Plant Cell Environ. 2006;29:1924-35.

93. Andersson MX, Stridh MH, Larsson KE, Liljenberg C, Sandelius AS. Phosphate-deficient oat replaces a major portion of the plasma membrane phospholipids with the galactolipid digalactosyl diacylglycerol. FEBS Lett. 2003;537:128-32. https://doi.org/10.1016/S0014-5793(03)00109-1.

94. Shimojima M, Watanabe T, Madoka Y, Koizumi R, Yamamoto MP, Masuda $K$, et al. Differential regulation of two types of monogalactosyl diacylglycerol synthase in membrane lipid remodelling under phosphatelimited conditions in sesame plants. Front Plant Sci. 2013;4:469. https:// doi.org/10.3389/fpls.2013.00469.

95. Hammond JP, White PJ. Sucrose transport in the phloem: Integrating root responses to phosphorus starvation. J Exp Bot. 2008:59:93-109.

96. Park MR, Baek SH, de Los Reyes BG, Yun SJ, Hasenstein KH. Transcriptome profiling characterizes phosphate deficiency effects on carbohydrate metabolism in rice leaves. J Plant Physiol. 2012;169:193-205. https://doi.org/10.1016/j.jplph.2011.09.002.

97. Rychter AM, Randall DD. The effect of phosphate deficiency on carbohydrate metabolism in bean roots. Physiol Plant. 1994;91:383-8.

98. Wanke M, Ciereszko I, Podbielkowska M, Rychter AM. Response to phosphate deficiency in bean (Phaseolus vulgaris L.) roots. Respiratory metabolism, sugar localization and changes in ultrastructure of bean root cells. Ann Bot. 1998:82:809-819.

99. Heldt HW, Chon CJ, Maronde D. Role of orthophosphate and other factors in the regulation of starch formation in leaves and isolated chloroplasts. Plant Physiol. 1977;59:1146-55.

100. Hermans C, Hammond JP, White PJ, Verbruggen N. How do plants respond to nutrient shortage by biomass allocation? Trends Plant Sci. 2006;11:610-7.

101. Fredeen AL, Raab TK, Rao IM, Terry N. Effects of phosphorus nutrition on photosynthesis in Glycine max L. Merr Planta. 1990;181:399-405.

102. Pieters AJ, Paul MJ, Lawlor DW. Low sink demand limits photosynthesis under Pi deficiency. J Exp Bot. 2001;52:1083-91.

103. Lauer MJ, Pallardy SG, Belvins DG, Randall DD. Whole leaf carbon exchange characteristics of phosphate deficient soybeans (Glycine max L.), Plant Physiol. 1989;91:848-854.

104. Abel S, Ticconi CA, Delatorre CA. Phosphate sensing in higher plants. Physiol Plant. 2002;115:1-8.

105. Jacob J, Lawlor DW. Stomatal and mesophyll limitations of photosynthesis in phosphate deficient sunflower, maize and wheat plants. J Exp Bot. 1991:42:1003-11.

106. Qiu I, Israel DW. Carbohydrate accumulation and utilization in soybean plants in response to altered phosphorus nutrition. Physiol Plant. 1994;90:722-8

107. Plaxton WC, Carswell MC. Metabolic aspects of the phosphate starvation response in plants. In: Lerner HR, editor. Plant Responses to Environmental Stresses: From Phytohormones to Genome Reorganization. New York: Marcel Dekker; 1999. p. 349-72. 
108. Amtmann A, Hammond JP, Armengaud P, White PJ. Nutrient sensing and signalling in plants: potassium and phosphorus. Adv Bot Res. 2005:43:209-57.

109. Benkova E, Michniewicz M, Sauer M, Teichmann T, Seifertova D, Jurgens $\mathrm{G}$, et al. Local, efflux-dependent auxin gradients as a common module for plant organ formation. Cell. 2003;1 15:591-602. https://doi.org/10. 1016/s0092-8674(03)00924-3.

110. Gonzalez-Mendoza V, Zurita-Silva A, Sanchez Calderon L, SanchezSandoval ME, Oropeza-Aburto A, et al. APSR1, a novel gene required for meristem maintenance is negatively regulated by low phosphate availability. Plant Sci. 2013;205:2-12.

111. Floss DS, Levy JG, Levesque-Tremblay V, Pumplin N, Harrison MJ. DELLA proteins regulate arbuscule formation in arbuscular mycorrhizal symbiosis. Proc Natl Acad Sci USA. 2013;110:5025-34.

112. Chen CY, Wu K, Schmidt W. The histone deacetylase HDA19 controls root cell elongation and modulates a subset of phosphate starvation responses in Arabidopsis. Sci Rep. 2015;5:15708.

113. Zahraeifard S, Foroozani M, Sepehri A, Oh D-H, Wang G, Mangu V, Chen B, Baisakh N, Dassanayake M, Smith AP. Rice H2A.Z negatively regulates genes responsive to nutrient starvation but promotes expression of key housekeeping genes. J Exp Bot. 2018;69:4907-4919.

114. Zhang Q, Oh D-H, DiTusa SF, RamanaRao MV, Baisakh N, Dassanayake M. Smith AP () Rice nucleosome patterns undergo remodeling coincident with stress-induced gene expression. BMC Genomics. 2018;19:97.
115. Yong-VillalobosL, Gonzalez-Morales SI, Wrobel K, Gutierrez-Alanis D, Cervantes-Perez SA, Hayano-Kanashiro C, et al. Methylome analysis reveals an important role for epigenetic changes in the regulation of the Arabidopsis response to phosphate starvation. Proc Natl Acad Sci USA. 2015;112:E7293-302.

116. Yong-Villalobos L, Cervantes-Pérez SA, Gutiérrez-Alanis D, Gonzáles Morales S, Martínez O, Herrera-Estrella L. Phosphate starvation induces DNA methylation in the vicinity of cis-acting elements known to regulate the expression of phosphate-responsive genes. Plant Signal Behav. 2016;11:1173300. https://doi.org/10.1080/15592324.2016.1173300.

117. Chin JH, Gamuyao R, Dalid C, Bustamam M, Prasetiyono J, Moeljopawiro $S$, et al. Developing rice with high yield under phosphorus deficiency: Pup1 sequence to application. Plant Physiol. 2011;156:1202-16.

\section{Publisher's Note}

Springer Nature remains neutral with regard to jurisdictional claims in published maps and institutional affiliations.
Ready to submit your research? Choose BMC and benefit from:

- fast, convenient online submission

- thorough peer review by experienced researchers in your field

- rapid publication on acceptance

- support for research data, including large and complex data types

- gold Open Access which fosters wider collaboration and increased citations

- maximum visibility for your research: over $100 \mathrm{M}$ website views per year

At BMC, research is always in progress.

Learn more biomedcentral.com/submissions 